\title{
UbiB proteins regulate cellular CoQ distribution
}

\author{
Zachary A. Kemmerer ${ }^{1,2,7}$, Kyle P. Robinson ${ }^{1,2,7}$, Jonathan M. Schmitz ${ }^{1,2}$, Brett R. Paulson ${ }^{4}$, Adam \\ Jochem $^{1,2}$, Paul D. Hutchins ${ }^{4}$, \& Joshua J. Coon ${ }^{3,4,5}$, and David J. Pagliarini ${ }^{1,2,6 *}$ \\ ${ }^{1}$ Morgridge Institute for Research, Madison, WI 53715, USA. \\ ${ }^{2}$ Department of Biochemistry, University of Wisconsin-Madison, Madison, WI 53706, USA. \\ ${ }^{3}$ Genome Center of Wisconsin, Madison, Wisconsin 53706, USA. \\ ${ }^{4}$ Department of Chemistry, University of Wisconsin-Madison, Madison, WI 53706, USA. \\ ${ }^{5}$ Department of Biomolecular Chemistry, University of Wisconsin-Madison, Madison, WI 53706, USA. \\ ${ }^{6}$ Departments of Cell Biology and Physiology; Biochemistry and Molecular Biophysics; and Genetics, \\ Washington University School of Medicine, St. Louis, MO 63110 \\ ${ }^{7}$ These authors contributed equally to this work. \\ *e-mail: pagliarini@wustl.edu
}

Abstract

Coenzyme $\mathbf{Q}(\mathrm{CoQ}$, ubiquinone) is a redox-active lipid essential for many core metabolic processes in mitochondria, including oxidative phosphorylation ${ }^{1-3}$.

While lesser appreciated, CoQ also serves as a key membrane-embedded

antioxidant throughout the cell ${ }^{4}$. However, how CoQ is mobilized from its site of

synthesis on the inner mitochondrial membrane to other sites of action remains a

reciprocally regulate this process. Loss of Cqd1 skews cellular CoQ distribution

stress caused by exogenous polyunsaturated fatty acids (PUFAs), whereas loss

30 auxiliary protein for CoQ biosynthesis. Overall, our results reveal new protein

31 machinery central to CoQ trafficking in yeast and lend new insights into the

32 broader interplay between mitochondrial and cellular processes. 


\section{Extramitochondrial CoQ combats oxidative stress}

34 To our knowledge, no proteins have yet been directly associated with cellular CoQ

35 trafficking from mitochondria, but the extreme hydrophobicity of $\mathrm{CoQ}$ suggests that this

36 process likely requires dedicated machinery. We sought to identify such proteins by

37 exploiting CoQ's extramitochondrial antioxidant role. Budding yeast (Saccharomyces

38 cerevisiae) lacking $\mathrm{CoQ}$ or phospholipid hydroperoxide glutathione peroxidases

39 (PHGPx) are sensitive to the oxidative stress conferred by exogenous polyunsaturated

40 fatty acids (PUFAs), such as linolenic acid (18:3) ${ }^{5,6}$. To force cells into relying more

41 heavily on the antioxidant properties of CoQ, we deleted all three PHGPx genes in

42 W303 S. cerevisiae ( $\Delta g p \times 1 \Delta g p \times 2 \Delta g p \times 3$, hereafter referred to as $\Delta g p \times 1 / 2 / 3)$. We

43 validated that this strain is sensitized to $18: 3$ treatment and demonstrated that this

44 sensitivity is dampened when cellular CoQ levels are augmented through

45 supplementation with the soluble CoQ precursor 4-hydroxybenzoate (4-HB) (Fig. 1a-b).

46 Importantly, the CoQ analog decylubiquinone was markedly more effective at protecting

47 against PUFA stress than its mitochondria-targeted counterpart, mitoquinone,

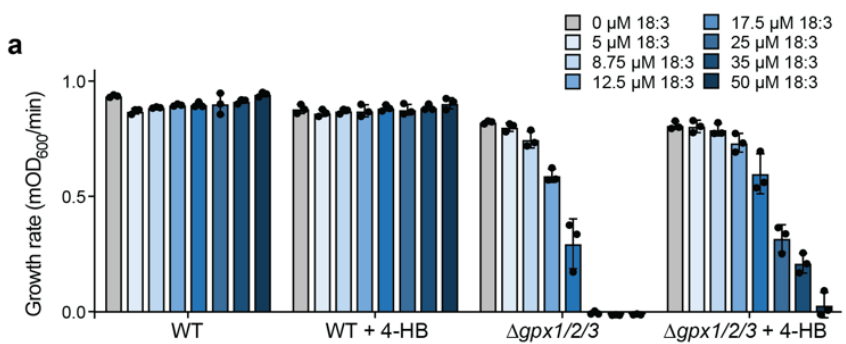

b

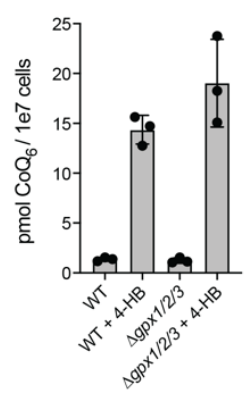

c

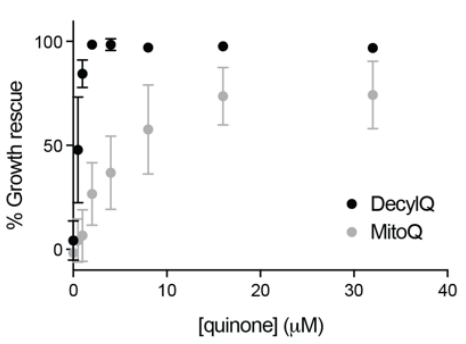

Fig. 1: Extramitochondrial CoQ combats oxidative stress. $a$, Growth rate of wild type (WT) and $\Delta g p \times 1 / 2 / 3$ yeast in synthetic complete media minus para-aminobenzoate (pABA-) containing $2 \%(\mathrm{w} / \mathrm{v})$ glucose (mean $\pm S D, n=3$ ) and the indicated additives. 4-hydroxybenzoate, 4-HB; linolenic acid (PUFA), 18:3. b, Total $\mathrm{CoQ}$ from WT and $\Delta g p x 1 / 2 / 3$ yeast described in a (mean $\pm S D, n=3)$. c, Rescue assay under the conditions described in a comparing the ability of decylubiquinone (DecylQ) and mitoquinone (MitoQ) to restore growth of $\Delta g p \times 1 / 2 / 3$ yeast treated with $35 \mu \mathrm{M}$ 18:3 (mean $\pm \mathrm{SD}, \mathrm{n}=3$ ). 
suggesting that extramitochondrial CoQ is the predominant mediator of PUFA

49 resistance (Fig. 1c). This is consistent with previous data showing that exogenous

50 PUFAs are incorporated into endogenous membranes slowly, and therefore, populate

51 non-mitochondrial membranes first ${ }^{6}$. Thus, we established a strain whose survival in the

52 presence of PUFAs is especially dependent on extramitochondrial CoQ.

\section{Loss of Cqd1 confers PUFA resistance}

55 We reasoned that suppressor mutations that increase extramitochondrial CoQ levels

56 would enhance PUFA resistance in the $\Delta g p \times 1 / 2 / 3$ strain, so we performed a forward-

57 genetic suppressor screen (Fig. 2a). We randomly mutagenized this strain with ethyl

58 methanesulfonate (EMS) and isolated colonies tolerant of 18:3 treatment. From $\sim 20,000$

59 unique mutant colonies, we obtained four hit strains with substantial PUFA resistance

60 (Fig. 2b). We then performed whole-genome sequencing that revealed non-

61 synonymous mutations in 442 unique genes across these four strains (Extended Data

62 Table 1). These mutants were ranked using PROVEAN (Protein Variation Effect

63 Analyzer), a software tool for predicting deleterious protein changes ${ }^{7}$. PROVEAN

64 assigns a disruption score (D-Score) that reflects the likelihood that a given mutation is

65 deleterious. In our collective dataset, 99 genes achieved a D-Score below the strict

66 threshold of -4.1 (Fig. 2c; Extended Data Table 1). Given the overall limited overlap in

67 hits between mutant strains, it is likely that our dataset includes multiple genes that

68 contribute to an enhanced PUFA resistance phenotype. 

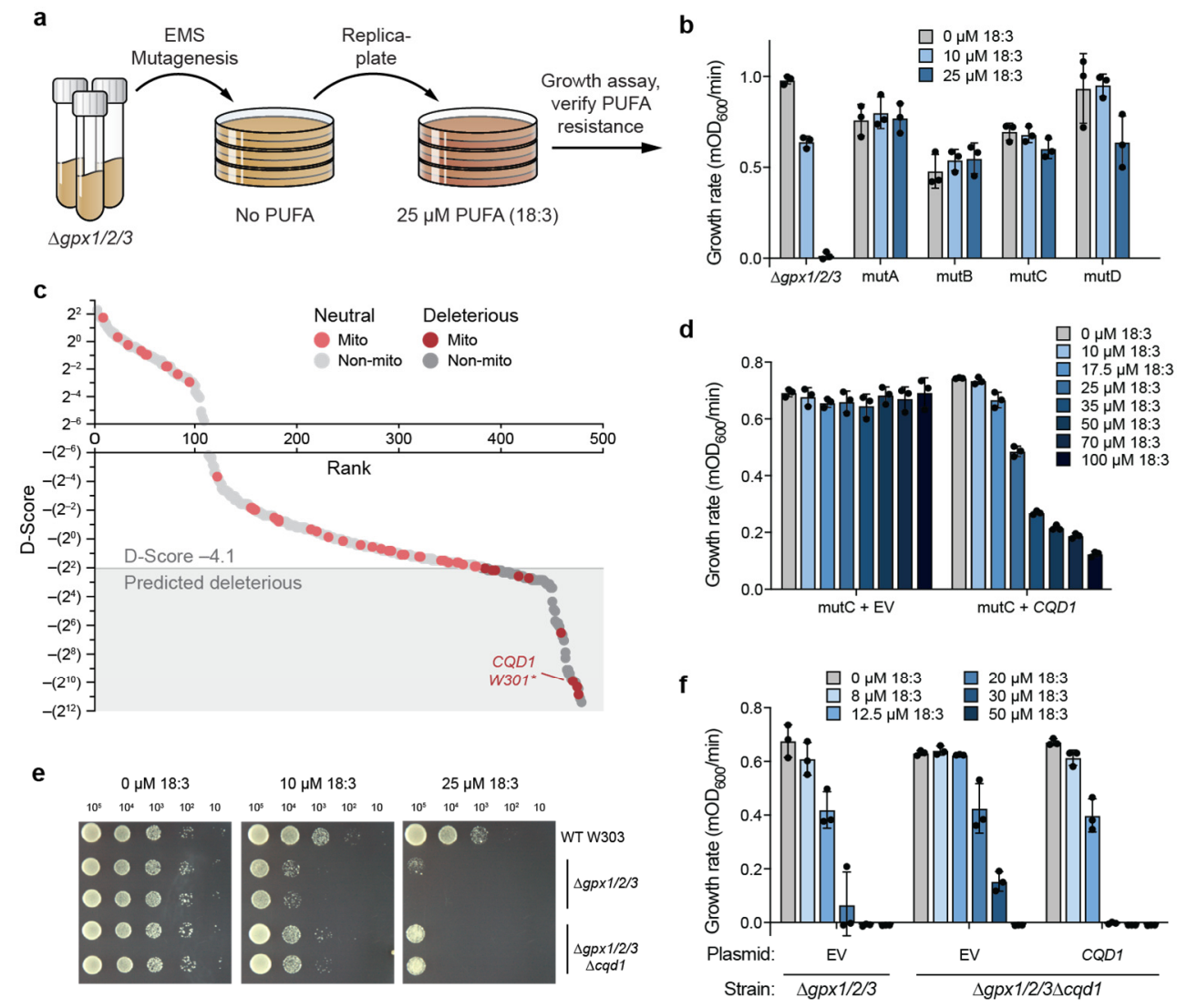

Fig. 2: Genome-wide screen for CoQ trafficking genes identifies uncharacterized UbiB protein Cqd1. a, Schematic of forward-genetic yeast screen for genes involved in CoQ trafficking. b. Growth rates of $\Delta g p \times 1 / 2 / 3$ and four mutant strains resistant to 18:3 treatment (mutA-D). Yeast were assayed in pABAmedia containing $2 \%(\mathrm{w} / \mathrm{v})$ glucose with $0-25 \mu \mathrm{M} 18: 3$ (mean $\pm S D, n=3)$. c, Mutant strains mutA-D were submitted for whole-genome sequencing to identify non-synonymous mutations (total $=442$ ). Mutations were analyzed with PROVEAN to filter for likely deleterious changes (D-score $\leq-4.1$, shaded box). Gray, all genes; red, mitochondrial genes. Light, predicted neutral; dark, predicted deleterious. d, Growth rate of mutC yeast expressing empty vector $(E V)$ or endogenous CQD1 (mean $\pm \mathrm{SD}, \mathrm{n}=3$ ). Yeast were assayed under the conditions described in b with 0-100 $\mu \mathrm{M}$ 18:3. e, Drop assay of WT, $\Delta g p \times 1 / 2 / 3$, and $\Delta g p \times 1 / 2 / 3 \Delta c q d 1$ yeast grown for 3 days on solid pABA- medium containing $2 \%(\mathrm{w} / \mathrm{v})$ glucose, $0.5 \%(\mathrm{w} / \mathrm{v})$ ethanol (EtOH), and 0-25 $\mu \mathrm{M} 18: 3$. f, Growth rates of $\Delta g p x 1 / 2 / 3$ and $\Delta g p \times 1 / 2 / 3 \Delta c q d 1$ yeast expressing $\mathrm{EV}$ or endogenous $C Q D 1$ (mean $\pm S D, n=3$ ). Yeast were assayed under the conditions described in $\mathbf{b}$ with 0-50 $\mu$ M 18:3. Source data for panel $\mathbf{c}$ is provided as a Source Data file.

We chose to focus on mitochondrial proteins for further examination since, to our

71 be identified. Of the nine mitochondrial proteins harboring likely deleterious mutations,

72 one, Ypl109c (renamed here as Cqd1, see below), is an uncharacterized protein that

73 resides on the inner mitochondrial membrane (IMM), making it an attractive candidate

74 for further study (Fig. 2c; Extended Data Fig. 1a). Moreover, Cqd1 possesses the same 
75 UbiB family atypical kinase/ATPase domain as Coq8, an essential protein for CoQ

76 synthesis that resides on the matrix face of the $\mathrm{IMM}^{8-11}$. Our recent work suggests that

77 Coq8 ATPase activity may be coupled to the extraction of hydrophobic CoQ precursors

78 from the IMM for subsequent processing by membrane-associated matrix enzymes ${ }^{12}$.

79 Cqd1 resides on the opposite side of the IMM, facing the intermembrane space s,13 $^{9}$

80 (Extended Data Fig. 1b), physically separated from the other CoQ-related enzymes but

81 still positioned for direct access to membrane-embedded CoQ precursors and mature

$82 \mathrm{CoQ}$. Furthermore, a recent study reported that haploinsufficiency of human CQD1

83 ortholog $A D C K 2$ led to aberrant mitochondrial lipid oxidation and myopathy associated

84 with CoQ deficiency ${ }^{14}$.

85 In our screen, mutant C (mutC) contains an early stop codon in CQD1 (Fig. 2c,

86 Extended Data Fig. 1c). To test whether this mutation is important for mutC's

87 phenotype, we reintroduced WT CQD1 into this strain under its endogenous promoter.

88 Indeed, this reintroduction re-conferred PUFA sensitivity (Fig. 2d). Furthermore, deletion

89 of $C Q D 1$ in the parent $\triangle g p \times 1 / 2 / 3$ strain, which lacks all other mutC mutations, was

90 sufficient to enhance PUFA resistance (Fig. 2e-f). Collectively, these data demonstrate

91 that disruption of CQD1 is at least partially causative for mutC's PUFA-resistant

92 phenotype.

\section{Cqd1 affects CoQ distribution}

95 Our results above suggest that loss of CQD1 confers cellular resistance to PUFA-

96 mediated oxidative stress by increasing extramitochondrial CoQ. We reasoned that this

97 was likely rooted either in a general increase in CoQ production or in its redistribution.

98 To test these models, we first measured total levels of CoQ and its early mitochondrial 
99 precursor polyprenyl-4-hydroxybenzoate (PPHB) in cells lacking CQD1 or control genes

100 (Fig. 3a-c). As expected, disruption of HFD1, which encodes the enzyme that produces

101 the soluble CoQ precursor 4-HB, led to loss of CoQ and PPHB, while disruption of

102 COQ8 caused complete loss of CoQ with the expected buildup of the PPHB precursor.

103 However, we found no significant change in CoQ or PPHB levels in the $\Delta c q d 1$ strain,

104 demonstrating that Cqd1 is essential neither for CoQ biosynthesis nor the import of CoQ 105 precursors under the conditions of our analyses.

106 To next examine CoQ distribution, we fractionated yeast and measured CoQ

107 levels (Fig. 3d; Extended Data Fig. 2a). We observed that $\Delta c q d 1$ yeast had a significant

108 increase in CoQ from the non-mitochondrial (NM) fraction, consisting of organelles and

109 membranes that do not pellet with mitochondria, and a corresponding decrease in

110 mitochondrial (M) CoQ. Deletion of the tricarboxylic acid (TCA) cycle enzyme Kgd1 had

111 no effect on relative CoQ levels (Fig. 3d) despite causing a deficiency in respiratory

112 growth (Fig. 3e), indicating that general mitochondrial dysfunction does not perturb CoQ

113 distribution. The increased extramitochondrial CoQ in $\Delta c q d 1$ yeast is consistent with the

114 observation that deleting CQD1 increases PUFA resistance (Fig. 2e-f).

115 To our knowledge, this is the first example of a genetic disruption leading to

116 altered cellular distribution of endogenous $\mathrm{CoQ}$, hence our renaming of this gene $\underline{\mathrm{C}} \underline{\mathrm{Q}}$

117 Distribution 1 (CQD1). To further validate this finding, we examined growth in glycerol, a

118 non-fermentable carbon source, which requires an intact mitochondrial electron

119 transport chain. We reasoned that a decrease in mitochondrial CoQ would disrupt

120 respiratory growth in media depleted of CoQ precursors. Indeed, deletion of CQD1

121 significantly reduced respiratory growth rate in this medium (Fig. 3e). To confirm that 
122 this defect is caused by CoQ depletion, we rescued growth with CoQ of different

123 isoprene tail lengths $\left(\mathrm{CoQ}_{2}\right.$ and $\left.\mathrm{CoQ}_{4}\right)$ and with $\mathrm{CoQ}$ precursors, which are more readily

124 delivered due to their solubility (Fig. 3f). Endogenous expression of CQD1 rescued

125 respiratory growth without affecting total CoQ levels (Fig. 3g, Extended Data Fig. 2b),

126 further supporting the hypothesis that $\mathrm{CoQ}$ distribution, not biosynthesis, is perturbed in

$127 \Delta c q d 1$ yeast.

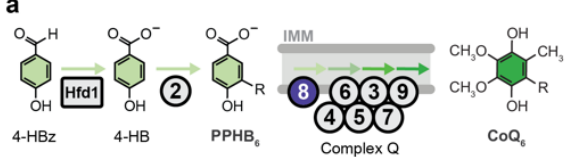

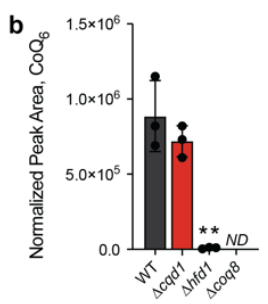
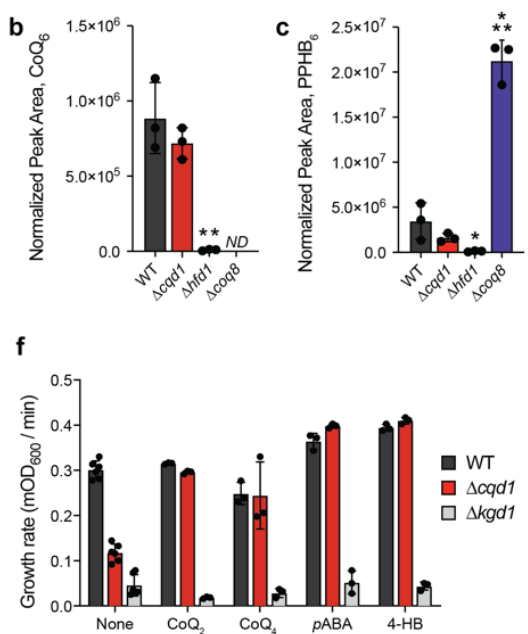
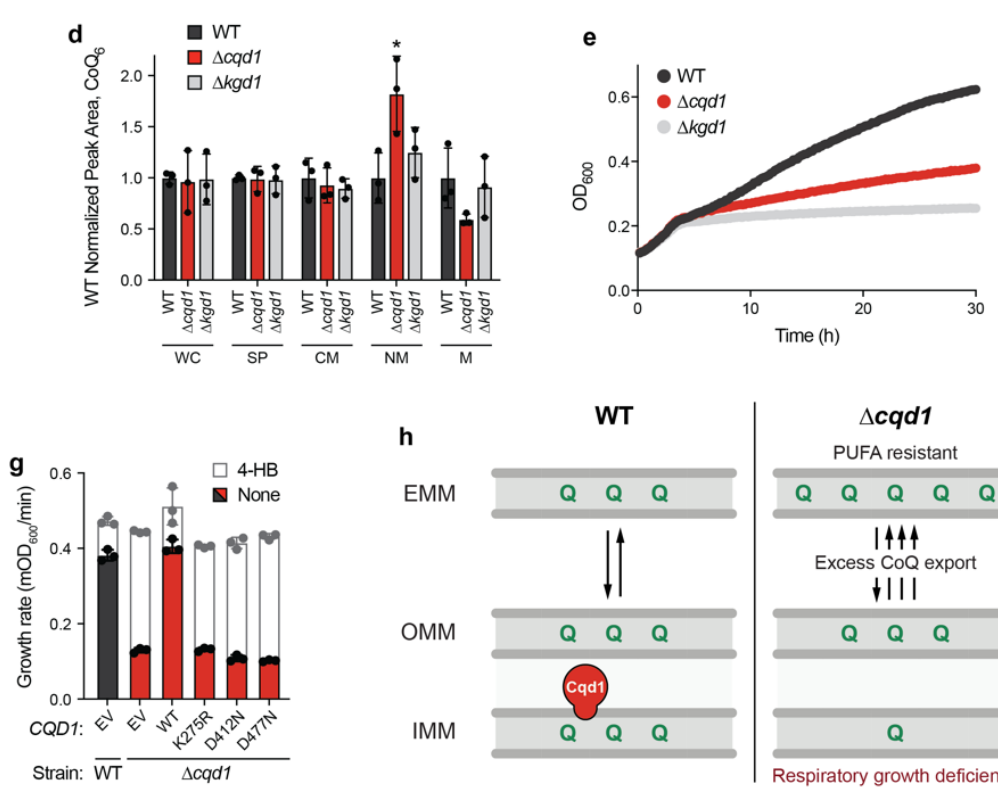
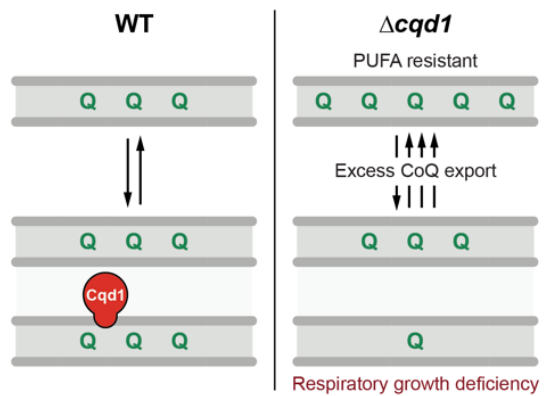

Fig. 3: Cqd1 influences cellular CoQ distribution. a, Schematic of CoQ biosynthesis pathway. Polyprenyl hydroxybenzoate (PPHB) is an early precursor that undergoes a series of head group modifications by IMM-associated Coq enzymes (complex Q) to produce CoQ. Hfd1 is essential for PPHB synthesis, while Coq8 is required for production of CoQ. b. Total CoQ and $\mathbf{c}$, polyprenyl-4-hydroxybenzoate (PPHB) from WT, $\Delta c q d 1, \Delta h f d 1$, and $\Delta c o q 8$ yeast (mean $\pm \mathrm{SD}, \mathrm{n}=3$ ); not detected, ND. d, CoQ from subcellular fractions derived from WT, $\Delta c q d 1$, and $\Delta k g d 1$ yeast (mean $\pm \mathrm{SD}, \mathrm{n}=3$ ). Spheroplast, SP; crude mitochondria, CM; non-mitochondrial fraction, NM; enriched mitochondria, M. e, Growth assay of WT, $\Delta c q d 1$, and $\Delta k g d 1$ yeast in pABA- media containing $0.1 \%(\mathrm{w} / \mathrm{v})$ glucose and $3 \%(\mathrm{w} / \mathrm{v})$ glycerol (mean, $\mathrm{n}=6)$. Yeast enter the respiratory phase of growth after $\sim 4$ hours in this growth condition. f, Growth rate of WT, $\Delta c q d 1$, and $\Delta k g d 1$ yeast assayed under conditions described in $d$ (mean $\pm S D$; none $n=6$, all others $n=3$ ). Yeast were grown in the presence and absence of $100 \mu \mathrm{M} \mathrm{CoQ}$ analogs $\left(\mathrm{CoQ}_{2}, \mathrm{CoQ}_{4}\right)$ and $1 \mu \mathrm{M}$ CoQ precursors (pABA, 4$\mathrm{HB}$ ). g, Growth rate of WT and $\triangle$ cqd1 yeast transformed with the indicated plasmids (EV, CQD1 or CQD1 point mutants) and grown in Ura-, pABA- media containing $0.1 \%(\mathrm{w} / \mathrm{v})$ glucose and $3 \%(\mathrm{w} / \mathrm{v})$ glycerol (mean $\pm \mathrm{SD}, \mathrm{n}=3$ ). Yeast were treated with 0 (colored bars) or $1 \mu \mathrm{M} 4-\mathrm{HB}$ (white bars, superimposed) to determine rescue of respiratory growth. $\mathbf{h}$, Model for Cqd1's putative role in cellular CoQ distribution. Significance calculated by a two-tailed Student's $t$-test; ${ }^{*}=p<0.05,{ }^{* *}=p<0.01,{ }^{* * *}=p<0.001$. 
130 COQ8A) revealed that it possesses an atypical protein kinase-like (PKL) fold that

131 endows ATPase activity but occludes larger proteinaceous substrates from entering the

132 active site ${ }^{11,15}$ (Extended Data Fig. 2c-e). Unlike COQ8, Cqd1 is recalcitrant to

133 recombinant protein purification; therefore, in lieu of direct in vitro activity assays, we

134 examined the ability of Cqd1 point mutants to rescue the respiratory growth defect of

$135 \Delta c q d 1$ yeast. Similar to $\mathrm{Coq} 8^{11,12,15}$, the ability of Cqd1 to rescue the $\Delta c q d 1$ respiratory

136 growth deficiency depended on core protein kinase-like $(P K L)$ family residues ${ }^{16}$ required

137 for phosphoryl transfer (Fig. 3g) and on quintessential UbiB motif residues (Extended

138 Data Fig. 2e-h). Further biochemical work is required to prove Cqd1's enzymatic

139 activity; however, these data support a model whereby Cqd1's ability to promote CoQ

140 distribution relies on atypical kinase/ATPase activity (Fig. 3h).

\section{Cqd2 counteracts Cqd1 function}

143 Beyond Coq8 and Cqd1, the S. cerevisiae genome encodes just one other member of

144 the UbiB family-YIr253w (aka Mcp2, and renamed here Cqd2). Cqd2 is also poorly

145 characterized and resides in the same location as Cqd1, on the outer face of the

$146 \operatorname{IMM}^{9,13,17}$ (Extended Data Fig. 1b). Previous studies have identified genetic and

147 physical interactions connecting Cqd2 to mitochondrial lipid homeostasis, but not to a

148 specific pathway ${ }^{17-19}$. Given the similarity between these three proteins (Extended Data

149 Fig. 2d-e), we anticipated that Cqd2 might also be connected to CoQ biology.

150 To test this hypothesis, we disrupted CQD2 in $\Delta g p x 1 / 2 / 3$ yeast and subjected

151 this strain to PUFA-mediated stress. Surprisingly, $\Delta g p x 1 / 2 / 3 \Delta c q d 2$ yeast exhibited an

152 enhanced sensitivity to PUFA treatment-the opposite phenotype to that of

$153 \Delta g p x 1 / 2 / 3 \Delta c q d 1$ (Fig. 4a; Extended Data Fig. 3a). Furthermore, $\Delta g p x 1 / 2 / 3 \Delta c q d 1 \Delta c q d 2$ 
154 yeast phenocopied the parental ( $\Delta g p x 1 / 2 / 3)$ strain (Fig. 4a; Extended Data Fig. 3a).

155 Under respiratory conditions, $\Delta c q d 2$ yeast exhibited no detectable change in growth.

156 However, deleting $C Q D 2$ from $\Delta c q d 1$ yeast $(\Delta c q d 1 \Delta c q d 2)$ restored this strain's impaired

157 respiratory growth rate to WT levels (Fig. 4b-c). Conversely, reintroduction of CQD2 into

a
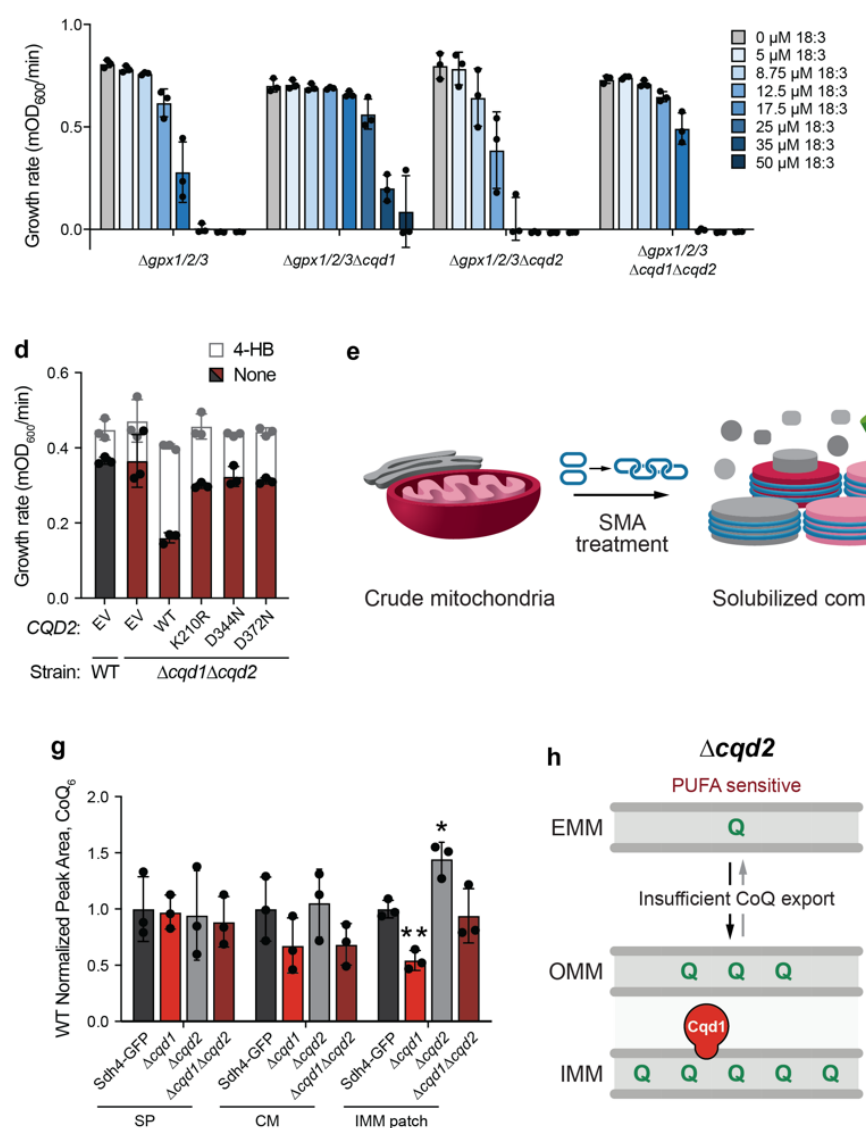

e

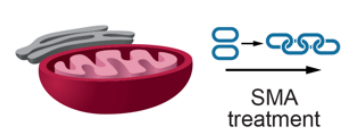

Crude mitochondria

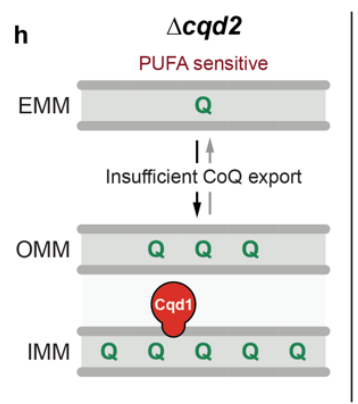

b

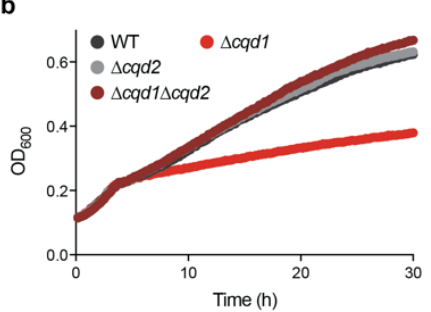

c

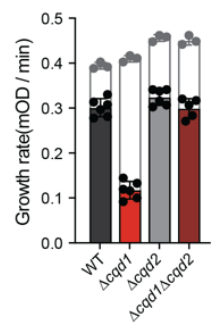

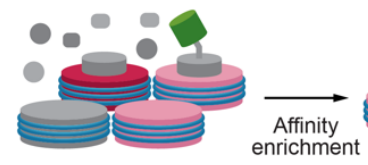
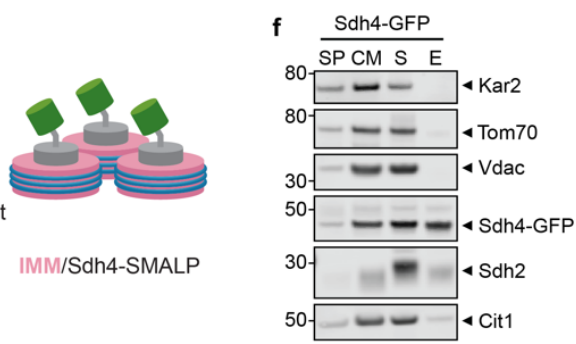

Solubilized components

IMM/Sdh4-SMALP
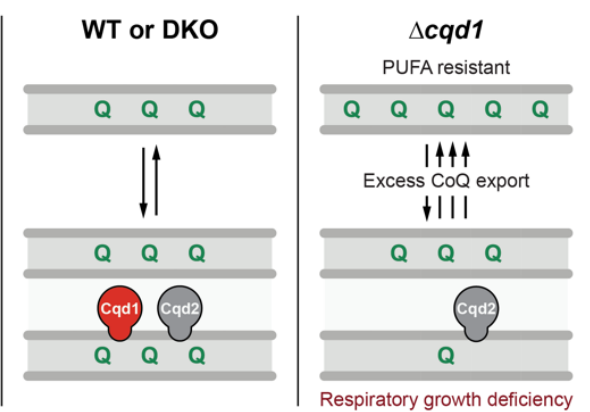

Fig. 4: Cqd2 function opposes Cqd1 control of CoQ distribution. a, Growth rate of $\Delta g p \times 1 / 2 / 3$ and the described yeast strains in $p A B A-$ media containing $2 \%(\mathrm{w} / \mathrm{v})$ glucose and the indicated additives (mean \pm $\mathrm{SD}, \mathrm{n}=3$ ). $\mathbf{b}$, Growth assay of WT, $\Delta c q d 1, \Delta c q d 2$, and $\Delta c q d 1 \Delta c q d 2$ yeast in $p A B A$ - media containing $0.1 \%$ $(\mathrm{w} / \mathrm{v})$ glucose and $3 \%(\mathrm{w} / \mathrm{v})$ glycerol (mean $\pm \mathrm{SD}, \mathrm{n}=6)$. c, Growth rate of yeast strains in $b$ treated with 0 (colored bars) or $1 \mu \mathrm{M} 4-\mathrm{HB}$ (white bars, superimposed) (mean $\pm \mathrm{SD}$; $0 \mu \mathrm{M} 4-\mathrm{HB} \mathrm{n}=6,1 \mu \mathrm{M} 4-\mathrm{HB} n=3$ ). d, Growth rate of WT and $\Delta c q d 1 \Delta c q d 2$ yeast transformed with the indicated plasmids (EV, CQD2 or CQD2 point mutants) and grown in Ura-, pABA- media containing $0.1 \%(\mathrm{w} / \mathrm{v})$ glucose and $3 \%(\mathrm{w} / \mathrm{v})$ glycerol (mean $\pm \mathrm{SD}, \mathrm{n}=3$ ). Yeast were treated with 0 (colored bars) or $1 \mu \mathrm{M} 4-\mathrm{HB}$ (white bars, superimposed) to determine recapitulation of respiratory growth defect. e, Schematic of Sdh4-GFP styrene maleic acid (SMA) lipid particle (SMALP) isolation. $\mathbf{f}$, Western blot to assess purity of SMALP isolation samples from endogenously tagged Sdh4-GFP yeast. Spheroplast, SP; crude mitochondria, CM; soluble, S; elution, E (or IMM patch). Kar2, endoplasmic reticulum; Tom70, outer mitochondrial membrane (OMM); Vdac, OMM; Sdh4-GFP, SMALP target/IMM; Sdh2, IMM; Cit1, mitochondrial matrix. g, CoQ from subcellular fractions derived from SMALP isolation described in $\mathbf{f}$ for the indicated strains (mean $\pm S D, n=3$ ). $\mathbf{h}$, Summary model depicting opposing roles for yeast $\mathrm{UbiB}$ family proteins in cellular distribution of CoQ. Significance calculated by a two-tailed Student's $t$-test; ${ }^{*}=p<0.05,{ }^{* *}=p<0.01,{ }^{* * *}=p<0.001$. 
158 the $\Delta c q d 1 \Delta c q d 2$ strain recapitulated the respiratory growth deficiency of $\Delta c q d 1$ (Fig.

159 4d). Total cellular CoQ levels remained unchanged (Extended Data Fig. 3b), again

160 suggesting these CoQ-related phenotypes are unrelated to CoQ biosynthesis. Similar to

161 Cqd1 (Fig. 3g), Cqd2 function was dependent on intact canonical PKL and UbiB-specific

162 residues (Fig. 4d, Extended Data Fig. 3c-e), suggesting that all three UbiB family

163 proteins in yeast are active phosphoryl transfer enzymes.

The analyses above, coupled with the submitochondrial location of Cqd1 and

165 Cqd2, suggest a model whereby these enzymes may reciprocally regulate the amount

166 of CoQ within the IMM. To test this directly, we used the amphipathic polymer styrene

167 maleic acid (SMA) to solubilize integral membrane proteins into detergent-free SMA

168 lipid particles (SMALPs) ${ }^{20}$ from yeast harboring an endogenously-tagged subunit of

169 mitochondrial complex II (Sdh4-GFP). We reasoned that purifying lipid patches

170 containing Sdh4, which directly interacts with CoQ to facilitate succinate dehydrogenase

171 (Complex II) activity ${ }^{21}$, would yield a suitable lipid microenvironment to measure IMM-

172 localized CoQ. After solubilization (Extended Data Fig. 3f), we isolated native IMM

173 patches that possessed Sdh4-GFP using a recombinantly purified His-tagged GFP

174 nanobody (Fig. 4e; Extended Data Fig. 3g-h). We show that purified Sdh4-GFP IMM

175 patches are largely void of extramitochondrial and outer mitochondrial membrane

176 (OMM) protein contamination (Fig. 4f), making this a reliable sample for assessing IMM

177 CoQ abundance.

178 We generated a panel of deletion strains in the Sdh4-GFP background to

179 investigate how loss of Cqd1 and Cqd2 impact CoQ abundance in this IMM

180 microenvironment. These yeast strains exhibited the same respiratory phenotypes as 
181 the W303 background strains and had similar levels of whole-cell CoQ (Extended Data

182 Fig. 3i-j). After solubilization and affinity enrichment (Extended Data Fig. 3k-I), Sdh4-

183 GFP IMM patch lipids were extracted for targeted CoQ measurements. Consistent with

184 our respiratory growth observations, $\Delta c q d 1$ yeast had significantly lower levels of IMM

185 patch CoQ. Conversely, the $\Delta c q d 2$ yeast had elevated IMM patch CoQ, while

$186 \Delta c q d 1 \Delta c q d 2$ yeast had levels similar to the parental strain (Fig. $4 \mathrm{~g}$ ). These data provide

187 direct evidence of protein-dependent changes in CoQ distribution, corroborating our

188 phenotypic observations. Taken together, our results suggest that Cqd1 and Cqd2

189 reciprocally regulate the levels of IMM CoQ and support a model wherein proper cellular

190 CoQ distribution is dependent on the balance of their activities (Fig. 4h).

192 Discussion

193 Our work demonstrates that two previously uncharacterized UbiB family proteins

194 influence the cellular distribution of mitochondria-derived CoQ. To our knowledge, Cqd1

195 and Cqd2 are the first proteins implicated in this process, which is essential for

196 providing membranes throughout the cell with the CoQ necessary for enzymatic

197 reactions and antioxidant defense. Further efforts are needed to establish how these

198 proteins support CoQ distribution mechanistically; however, their similarity to Coq8 and

199 the requirement for canonical PKL residues in their active sites suggests that Cqd1 and

200 Cqd2 may couple ATPase activity to the selective extraction/deposition of CoQ from/to

201 the IMM.

202 Once extracted from the IMM, we expect that subsequent steps would be

203 required to deliver CoQ throughout the cell. The multimeric ER-mitochondrial encounter

204 structure (ERMES) and mitochondrial contact site and cristae organizing system 
205 (MICOS) complexes facilitate interorganellar lipid and metabolite transfer ${ }^{22,23}$. Recent

206 work has revealed that CoQ biosynthetic machinery and MICOS subcomplexes often

207 colocalize with ERMES ${ }^{24-26}$, suggesting that these sites could serve as conduits for CoQ

208 transport. Additionally, COQ9 is a lipid binding protein that likely delivers CoQ

209 precursors to matrix enzymes ${ }^{27}$, suggesting that other lipid binding proteins may exist to

210 shuttle CoQ from mitochondria to other membranes. Our genetic screen has nominated

211 several extramitochondrial and cytosolic proteins as promising leads for these

212 processes.

213 Our investigations here focused on CoQ; however, it is possible that Cqd1 and

214 Cqd2 (aka Mcp2) influence lipid transport and homeostasis more broadly. Previous

215 work has identified an array of genetic interactions for Cqd1 and Cqd2 with lipid

216 biosynthesis and homeostasis genes ${ }^{19,28}$. Moreover, Cqd2 was previously identified as

217 a high-copy suppressor of a growth defect caused by loss of the ERMES subunit

218 Mdm1017. More recently, three conserved Cqd2 active site residues were shown to

219 mitigate rescue of $\Delta m d m 10$ yeast growth ${ }^{18}$, results that we confirm (Cqd2 K210R) and

220 expand upon with six additional residue mutations.

221 Finally, UbiB family proteins are found across all domains of life ${ }^{29}$. UbiB

222 homologs in plants (termed $\mathrm{ABC} 1 \mathrm{~K}$ proteins) are abundant, with 17 found in

223 Arabidopsis ${ }^{30}$. Many of these $\mathrm{ABC} 1 \mathrm{~K}$ proteins are localized to plastoglobules_plastid-

224 localized lipoprotein particles that contain various lipid-derived metabolites_and recent

225 work suggests that $\mathrm{ABC} 1 \mathrm{~K} 1$ and $\mathrm{ABC} 1 \mathrm{~K} 3$ may affect the mobility and exchange of their

226 subcellular plastoquinone-9 pools ${ }^{31}$, suggesting UbiB proteins might function in quinone

227 distribution across species. In humans, five UbiB proteins have been identified, ADCK1- 
5. While COQ8A (ADCK3) and COQ8B (ADCK4) have established roles in CoQ biosynthesis and human disease ${ }^{11,32,33}$, the biological roles of other ADCK proteins

230 remain elusive. Genome-wide knockdown studies have implicated these

231 uncharacterized $A D C K$ genes in several cancer disease states ${ }^{34-37}$. As novel targets for

232 human disease intervention, it will be important to determine if functional conservation

233 exists between Cqd1 and Cqd2 and their putative human orthologs, ADCK2 and

234 ADCK $1 / 5$, respectively. Recently, a crucial new role for extramitochondrial CoQ was

235 identified in mitigating ferroptosis, a type of cell death stemming from a buildup of toxic

236 lipid peroxides, suggesting that manipulating CoQ distribution could provide therapeutic

237 benefits ${ }^{38,39}$. Notably, we have developed small-molecule modulators for Coq $8^{12}$ and

$238 \mathrm{COQ}^{4} \mathrm{~A}^{40}$, indicating that $\mathrm{UbiB}$ proteins are promising druggable targets.

239 Collectively, our work to de-orphanize these poorly characterized mitochondrial

240 proteins represents the first step in addressing enduring questions regarding

241 endogenous cellular CoQ distribution and unlocking the therapeutic potential of

242 manipulating this pathway.

245 Yeast Strains and Cultures

246 Unless otherwise described, Saccharomyces cerevisiae haploid W303 (MATa his3 leu2

247 met15 trp1 ura3) yeast were used. For SMA-derived lipid nanodisc work, endogenous

248 GFP-tagged BY4741 (MATa his3 $\Delta 1$ leu2 $\Delta 0$ met15 $\Delta 0$ ura3 $\Delta 0$ ) yeast strains ${ }^{41}$ were

249 used. Yeast deletion strains were generated using standard homologous recombination

250 or CRISPR-mediated methods. For homologous recombination, open reading frames 
251 were replaced with the KanMX6, HygMX6, or NatMX6 cassette as previously

252 described ${ }^{42}$. Cassette insertion was confirmed by a PCR assay and DNA sequencing.

253 CRISPR-mediated deletions were performed as described $\mathrm{in}^{43} \cdot 20$-mer guide

254 sequences were designed with the ATUM CRISPR gRNA design tool

255 (https://www.atum.bio/eCommerce/cas9/input) and cloned into pRCC-K, and 500 ng of

256 the guide-inserted pRCC-K was used per yeast transformation. Donor DNA was 300

257 pmol of an 80-nt Ultramer consisting of $40 \mathrm{bp}$ upstream and $40 \mathrm{bp}$ downstream of the

258 ORF (for scarless deletions) or $\sim 6 \mu \mathrm{g}$ of PCR-amplified Longtine cassette with flanking

259 homology 40 bp upstream and 40 bp downstream of the ORF (for cassette-replacement

260 deletions).

262 Synthetic complete (and dropout) media contained drop-out mix (US Biological), yeast

263 nitrogen base (with ammonium sulfate and without amino acids) (US Biological), and

264 the indicated carbon source. $\mathrm{pABA}^{-}$(and dropout) media contained Complete

265 Supplement Mixture (Formedium), Yeast Nitrogen Base without amino acids and

266 without pABA (Formedium), and the indicated carbon source. All media were sterilized

267 by filtration $(0.22 \mu \mathrm{m}$ pore size $)$.

269 Yeast Growth Assay and Drop Assay

270 PUFA Growth Assays

271 To assay yeast growth in liquid media, individual colonies were used to inoculate

272 synthetic complete (or synthetic complete dropout) media ( $2 \%$ glucose, w/v) starter

273 cultures, which were incubated overnight $\left(30^{\circ} \mathrm{C}, 230 \mathrm{rpm}\right)$. Yeast were diluted to 
$2741.1 \times 10^{6}$ cells $/ \mathrm{mL}$ in $p \mathrm{ABA}^{-}$(or $p \mathrm{ABA}^{-}$dropout) media ( $2 \%$ glucose, w/v) and incubated

275 until early log phase $\left(30^{\circ} \mathrm{C}, 7-8 \mathrm{~h}, 230 \mathrm{rpm}\right)$. Yeast were swapped into fresh

276 pABA $^{-}$media ( $2 \%$ glucose, w/v) at an initial density of $5 \times 10^{6}$ cells $/ \mathrm{mL}$ with indicated

277 additives. The cultures were incubated $\left(30^{\circ} \mathrm{C}, 1140 \mathrm{rpm}\right)$ in an Epoch2 ${ }^{\mathrm{TM}}$ plate reader

$278\left(\right.$ BioTek $\left.^{\circledR}\right)$ in a sterile 96 well polystyrene round bottom microwell plate (Thermo) with a

279 Breathe-Easy ${ }^{\circledR}$ cover seal (Diversified Biotech). Optical density readings (A600) were

280 obtained every 10 minutes, and growth rates were calculated with Gen5 v3.02.2

281 software $\left(\right.$ BioTek $\left.^{\circledR}\right)$, excluding timepoints from stationary phase.

284 Individual colonies of S. cerevisiae were used to inoculate synthetic complete media $285\left(2 \%\right.$ glucose, w/v) starter cultures, which were incubated overnight $\left(30{ }^{\circ} \mathrm{C}, 230 \mathrm{rpm}\right)$. 286 For transformed yeast strains, the corresponding Ura- media was used. Yeast were 287 diluted to $1 \times 10^{6}-1.33 \times 10^{6}$ cells $/ \mathrm{mL}$ in $p A B A^{-}$media ( $2 \%$ glucose, w/v) and incubated 288 until early log phase $\left(30^{\circ} \mathrm{C}, 7-8 \mathrm{~h}, 230 \mathrm{rpm}\right)$. Yeast were swapped into $\mathrm{pABA}^{-}$media 289 with glucose $(0.1 \%, w / v)$ and glycerol $(3 \%, w / v)$ at an initial density of $5 \times 10^{6}$ cells $/ \mathrm{mL}$ 290 with indicated additives. The cultures were incubated $\left(30^{\circ} \mathrm{C}, 1140 \mathrm{rpm}\right)$ in an Epoch2 291 plate reader (BioTek) in a sterile 96 well polystyrene round bottom microwell plate 292 (Thermo) with a Breathe-Easy cover seal (Diversified Biotech). Optical density readings 293 (A600) were obtained every 10 minutes, and growth rates were calculated with Gen5 294 v3.02.2 software (BioTek), excluding timepoints before the diauxic shift and during 295 stationary phase growth. 


\section{Drop Assays}

298 Individual colonies of yeast were used to inoculate $p A B A$-limited media (2\% w/v

299 glucose, $100 \mathrm{nM}$ pABA $)$ starter cultures, which were incubated overnight $\left(30^{\circ} \mathrm{C}\right.$,

$300230 \mathrm{rpm})$. Cells were spun down (21,000 x g, $2 \mathrm{~min})$ and resuspended in water. Serial

301 dilutions of yeast $\left(10^{5}, 10^{4}, 10^{3}, 10^{2}\right.$, or 10 cells $)$ were dropped onto $p A^{-} A^{-}$media $(2 \%$

302 glucose and $1 \% \mathrm{EtOH}, \mathrm{w} / \mathrm{v})$ agar plates with indicated additives and incubated $\left(30^{\circ} \mathrm{C}\right.$, $303 \quad 2-3 d)$.

\section{Forward-genetic Screen}

306 Individual colonies of $\Delta g p \times 1 / 2 / 3$ yeast were used to inoculate YEPD starter cultures, 307 which were incubated overnight. $1.0 \times 10^{8}$ cells were pelleted, washed once with sterile 308 water, and resuspended in $2.5 \mathrm{~mL}$ of $100 \mathrm{mM}$ sodium phosphate buffer, $\mathrm{pH}$ 7.0. Ethyl 309 methanesulfonate (EMS) $(80 \mu \mathrm{L})$ was added, and cells were incubated $\left(90 \mathrm{~min}, 30^{\circ} \mathrm{C}\right.$, $310230 \mathrm{rpm})$. Cells were washed thrice with sodium thiosulfate $(5 \% \mathrm{w} / \mathrm{v})$ to inactivate EMS.

311 Cells were resuspended in water, and $1.0 \times 10^{4}$ cells were plated on pABA-limited $(2 \%$

312 w/v glucose, 100 nM pABA) agar plates. After 3 days, cells were replica-plated onto

$313 \operatorname{pABA}^{-}(2 \%$ glucose, w/v) plates with $0 \mu \mathrm{M}$ or $25 \mu \mathrm{M}$ linolenic acid (C18:3, Sigma).

314 Colonies that grew on $25 \mu \mathrm{M}$ linolenic acid were picked into YEPD overnight cultures

315 and struck on YEPD plates, and PUFA resistance phenotypes were confirmed with

316 plate reader growth assays. For mutant strains that grew in the presence of $25 \mu \mathrm{M}$

317 linolenic acid, genomic DNA was isolated with the MasterPure ${ }^{\mathrm{TM}}$ Yeast DNA Purification

318 Kit (Lucigen) and submitted to GENEWIZ for whole-genome sequencing. S. cerevisiae

319 genome assembly and variation calling were performed with SeqMan NGen 14 and 
ArrayStar 14 (DNASTAR Lasergene suite). Variant D-Score predictions were obtained

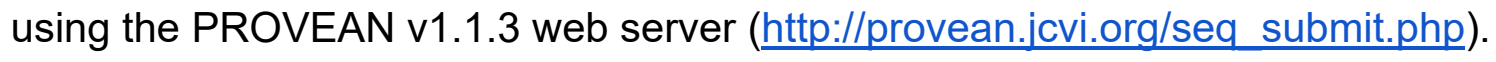

\section{Plasmid Cloning}

326 Expression plasmids were cloned with standard restriction enzyme cloning methods.

327 ORF specific primers were used to amplify Cqd1 (Ypl109c) and Cqd2 (Ylr253w) from

328 W303 yeast genomic DNA. Amplicons were treated with Dpnl to degrade genomic DNA

329 and ligated into the digested p416 GPD plasmid (Addgene). Cloning products were then

330 transformed into E. coli $10 \mathrm{G}$ chemically competent cells (Lucigen). Plasmids were

331 isolated from transformants and Sanger sequencing was used to identify those

332 containing the correct insertion.

334 Constructs containing Cqd1 and Cqd2 were digested with Sall and BamHI or HindIII to

335 liberate the GPD promoter. Digested backbones were then combined with amplified

336 endogenous promoter regions (1000 bases upstream for Cqd1, 500 bases upstream for

$337 \mathrm{Cqd} 2$ ) and ligated to generate endogenous promoter vectors for Cqd1 and Cqd2.

\section{Site-Directed Mutagenesis}

340 Point mutants were constructed as described in the Q $5^{\circledR}$ Site-Directed Mutagenesis Kit

341 (New England Biolabs) and were confirmed via Sanger sequencing. Yeast were

342 transformed as previously described ${ }^{44}$ with plasmids encoding $\mathrm{Cqd} 1$ and $\mathrm{Cqd} 2$ variants 
343 with their endogenous promoters and grown on uracil drop-out (Ura-) synthetic media

344 plates containing glucose $(2 \%, w / v)$.

\section{Homology Model Generation}

347 Amino acid sequences of Cqd1 and Cqd2 were threaded through COQ8A apo crystal 348 structure (PDB:4PED) via the online iTASSER webserver ${ }^{45}$. Superimposed homology 349 models were visualized in the PyMOL Molecular Graphics System (Version 2.0,

350 Schrödinger, LLC). Color schemes depicting protein domain organization were chosen 351 according to previous work ${ }^{15}$.

\section{Subcellular Fractionation}

354 Individual colonies of S. cerevisiae were used to inoculate synthetic complete media $355\left(2 \%\right.$ glucose, w/v) starter cultures, which were incubated overnight $\left(30^{\circ} \mathrm{C}, 230 \mathrm{rpm}\right)$.

356 Yeast were diluted to $1.2 \times 10^{6}$ cells $/ \mathrm{mL}$ in $50 \mathrm{~mL} p A B A^{-}$media ( $2 \%$ glucose, w/v) and

357 incubated until early log phase $\left(30^{\circ} \mathrm{C}, 12 \mathrm{~h}, 230 \mathrm{rpm}\right)$. Yeast were swapped into $2 \mathrm{~L}$ of $358 \quad \mathrm{pABA}^{-}$media with glucose $(0.1 \%, \mathrm{w} / \mathrm{v})$ and glycerol $(3 \%, \mathrm{w} / \mathrm{v})$ at an initial density of

$3595 \times 10^{4}$ cells $/ \mathrm{mL}$ and incubated until early log phase $\left(30^{\circ} \mathrm{C}, 20 \mathrm{~h}, 230 \mathrm{rpm}\right) .1 \times 10^{8}$ cells

360 were collected for whole-cell (WC) analyses. The remaining culture was pelleted by

361 centrifugation $(4,500 \times \mathrm{g}, 7 \mathrm{~min})$ and weighed $(5-6 \mathrm{~g})$. Pellets were then fractionated

362 using previously described methods ${ }^{46}$. To isolate crude mitochondria, samples were

363 pelleted by centrifugation $\left(15,000 \times \mathrm{g}, 10 \mathrm{~min}, 4^{\circ} \mathrm{C}\right)$. Crude mitochondria were

364 resuspended in SEM buffer (10 mM MOPS/KOH pH 7.2, 250 mM sucrose, 1 mM EDTA)

365 containing $10 \mu \mathrm{g}$ trypsin (sequencing grade, Promega) and rotated end-over-end 
overnight $\left(12 \mathrm{~h}, 4^{\circ} \mathrm{C}\right)$ to disrupt proteinaceous organelle contact tethers ${ }^{47}$. Digested samples were pelleted by centrifugation $\left(12,000 \times \mathrm{g}, 10 \mathrm{~min}, 4^{\circ} \mathrm{C}\right)$ and the supernatant was collected. Pelleted material was resuspended in $900 \mu \mathrm{L}$ SEM buffer containing 1 mM phenylmethylsulfonyl fluoride (SEM+PMSF) to deactivate trypsin. Resuspended material was pelleted $\left(12,000 \times \mathrm{g}, 10 \mathrm{~min}, 4^{\circ} \mathrm{C}\right)$ and the supernatant was collected. This was repeated once more and supernatant material was pooled $(2.7 \mathrm{~mL})$. To this, SEM buffer was added up to $10 \mathrm{~mL}$ before ultracentrifugation $\left(106,000 \times \mathrm{g}, 1 \mathrm{~h}, 4^{\circ} \mathrm{C}\right)$ to

373 collect microsomes (non-mitochondrial fraction; NM). Pelleted crude mitochondria were

374 resuspended in $700 \mu \mathrm{L}$ SEM+PMSF and then added to a freshly prepared sucrose

375 gradient (bottom to top: $1.5 \mathrm{~mL} 60 \%$ sucrose, $4 \mathrm{~mL} 32 \%$ sucrose, $1.5 \mathrm{~mL} 23 \%$ sucrose,

376 and $1.5 \mathrm{~mL} 15 \%$ sucrose) for separation by ultracentrifugation $\left(134,000 \times \mathrm{g}, 1 \mathrm{~h}, 4^{\circ} \mathrm{C}\right)$.

377 Enriched mitochondrial samples were recovered at the $32-60 \%$ interface and diluted

378 with $30 \mathrm{~mL}$ SEM. Mitochondria were pelleted $\left(15,000 \times \mathrm{g}, 10 \mathrm{~min}, 4^{\circ} \mathrm{C}\right)$ and

379 resuspended in fresh SEM $(150 \mu \mathrm{L}$ total). The protein concentration of all subcellular

380 fractions (spheroplasts, SP; crude mitochondria, CM; non-mitochondrial fraction, NM;

381 enriched mitochondria, M) was determined using the Pierce ${ }^{\mathrm{TM}}$ BCA Protein Assay Kit

382 (Thermo) before Western blot $(5 \mu \mathrm{g})$ analyses and lipid extractions.

384 GFP Nanobody

385 Recombinant Purification

386 pCA528-His-SUMO-GFP nanobody (GFPnb) constructs were transformed into RIPL

387 competent E. coli cells for protein expression. GFPnb was overexpressed in E. coli by

388 autoinduction overnight ${ }^{48}\left(37^{\circ} \mathrm{C}, 4 \mathrm{~h} ; 20^{\circ} \mathrm{C}, 20 \mathrm{~h}\right)$. Cells were isolated by centrifugation 
$389(4,500 \times g, 12 \mathrm{~min}, \mathrm{RT})$, flash frozen in $\mathrm{N}_{2}(\mathrm{I})$ dropwise, and stored at $-80{ }^{\circ} \mathrm{C}$. For protein

390 purification, cells were added to a Retsch ${ }^{\circledR}$ mixer mill MM 400 screw-top grinding jar

391 pre-equilibrated with $\mathrm{N}_{2}(\mathrm{I})$. The cells were lysed by cryogenic grinding $\left(-196{ }^{\circ} \mathrm{C}, 30 \mathrm{~Hz}\right.$,

$392120 \mathrm{~s} \times 3$ ). Ground cell pellet was collected and resuspended end-over-end for $1 \mathrm{~h}$ in

393 lysis buffer (160 mM HEPES pH 7.5, 400 mM NaCl, 0.25 mM PMSF, 1 Roche

394 cOmplete ${ }^{\mathrm{TM}}$ Protease Inhibitor Cocktail tablet, $500 \mathrm{U}$ Benzonase ${ }^{\circledR}$ Nuclease) at $4{ }^{\circ} \mathrm{C}$.

395 The lysate was clarified by centrifugation $\left(15,000 \times \mathrm{g}, 30 \mathrm{~min}, 4^{\circ} \mathrm{C}\right)$. Clarified lysate was

396 added to pre-equilibrated $\operatorname{TALON}^{\circledR}$ cobalt resin (Takara Bio) and incubated end-over-

397 end for $1 \mathrm{~h}$ at $4{ }^{\circ} \mathrm{C}$. TALON ${ }^{\circledR}$ resin was pelleted by centrifugation $\left(700 \times \mathrm{g}, 2 \mathrm{~min}, 4^{\circ} \mathrm{C}\right)$

398 and washed twice with equilibration buffer (160 mM HEPES pH 7.5, $400 \mathrm{mM} \mathrm{NaCl}, 0.25$

399 mM PMSF) and twice with wash buffer (160 mM HEPES pH 7.5, 400 mM NaCl, 0.25

400 mM PMSF, 20 mM imidazole). His-tagged protein was eluted with elution buffer (160

401 mM HEPES (pH 7.5), 400 mM NaCl, 0.25 mM PMSF, 400 mM imidazole). The eluted

402 protein was concentrated to $\sim 600 \mu \mathrm{L}$ with an Amicon ${ }^{\circledR}$ Ultra Centrifugal Filter (10 kDa

403 MWCO) and exchanged into equilibration buffer. Concentrated protein elution was

404 centrifuged $\left(15,000 \times \mathrm{g}, 5 \mathrm{~min}, 4^{\circ} \mathrm{C}\right)$ to pellet precipitate and filtered through a $0.22 \mu \mathrm{M}$

405 syringe filter. Concentrated protein elution was separated via size exclusion

406 chromatography on a HiLoad ${ }^{\mathrm{TM}} 16 / 600$ Superdex $^{\mathrm{TM}} 75$ pg. Fractions from the size

407 exclusion chromatography were analyzed by SDS-PAGE, and the fractions containing

408 GFPnb were pooled and concentrated to $\sim 1 \mathrm{~mL}$. The concentration of GFPnb was

409 determined by Bradford assay (Bio-Rad Protein Assay Kit II) and was diluted with

410 equilibration buffer and glycerol to a final concentration of $20 \mathrm{mg} / \mathrm{mL}$ protein (160 mM

411 HEPES pH 7.5, $400 \mathrm{mM} \mathrm{NaCl}, 10 \%$ glycerol). The final protein was aliquoted, flash 
412 frozen in $\mathrm{N}_{2}(\mathrm{I})$ and stored at $-80{ }^{\circ} \mathrm{C}$. Fractions from the protein preparation were

413 analyzed by SDS-PAGE.

414

415 Differential Scanning Fluorimetry

416 The differential scanning fluorimetry method (thermal shift assay) was performed as

417 described previously ${ }^{49}$. Purified recombinant GFPnb was diluted to a final concentration

418 of $4 \mu \mathrm{M}$ with DSF buffer (100 mM HEPES pH 7.5, 150mM NaCl) and 1:1250 SYPRO ${ }^{\circledR}$

419 Orange Dye (Life Tech). Thermal shift data was collected with QuantStudio Real-Time

420 PCR v1.2 software and analyzed with Protein Thermal Shift v1.3 software.

\section{Native Nanodisc Isolation}

423 Individual colonies of S. cerevisiae (BY4741) were used to inoculate synthetic

424 complete media ( $2 \%$ glucose, w/v) starter cultures, which were incubated overnight (30

$\left.425{ }^{\circ} \mathrm{C}, 230 \mathrm{rpm}\right)$. Yeast were diluted to $5 \times 10^{6} \mathrm{cells} / \mathrm{mL}$ in $50 \mathrm{~mL}$ pABA- media (2\% glucose,

$426 \mathrm{w} / \mathrm{v})$ and incubated until late log phase $\left(30^{\circ} \mathrm{C}, 16 \mathrm{~h}, 230 \mathrm{rpm}\right)$. Yeast were swapped

427 into $2 \mathrm{~L}_{\text {of }} p \mathrm{ABA}^{-}$media with glucose $(0.1 \%, w / v)$ and glycerol $(3 \%, w / v)$ at an initial

428 density of $2.5 \times 10^{6}$ cells $/ \mathrm{mL}$ and incubated until early log phase $\left(30^{\circ} \mathrm{C}, 16 \mathrm{~h}, 230 \mathrm{rpm}\right)$.

429 Yeast cultures were pelleted by centrifugation (4,500 x g, $7 \mathrm{~min})$ and weighed (2-3 g).

430 Pellets were then fractionated using previously described methods ${ }^{46}$. For preparative

431 scale affinity purification, crude mitochondria were resuspended in $50 \mu \mathrm{L} \mathrm{BB7.4} \mathrm{(0.6} \mathrm{M}$

432 sorbitol, 20 mM HEPES-KOH pH 7.4), diluted in $950 \mu \mathrm{L}$ ice cold BB7.S (20 mM HEPES-

$433 \mathrm{KOH} \mathrm{pH} \mathrm{7.4),} \mathrm{vortexed} \mathrm{for} 10 \mathrm{sec}$ (medium setting 8, Vortex Genie), and incubated on

434 ice for 30 minutes. Swollen mitochondria were then sonicated briefly (1/8" tip, 20\% 
435 amplitude) for 2 - 5 second pulses with 60 seconds between pulses. Mitoplasts with osmotically ruptured outer membranes were recovered by centrifugation at $(20,000 \times g$,

$43710 \mathrm{~min}, 4^{\circ} \mathrm{C}$ ). After removing the supernatant, each pellet was resuspended with $1 \mathrm{~mL}$

438 of Buffer B (20 mM HEPES-KOH pH 8.0, $200 \mathrm{mM} \mathrm{NaCl})$ containing 2\% (w/v) styrene

439 maleic acid copolymer (SMA, Polyscope SMALP ${ }^{\circledR} 25010 \mathrm{P}$ ) by repeat pipetting and

440 rotated end-over-end $\left(4 \mathrm{~h}, 4^{\circ} \mathrm{C}\right)$. Soluble SMA extracts were separated from non-

441 extracted material by centrifugation at $21,000 \times g$ for $10 \mathrm{~min}$ at $4{ }^{\circ} \mathrm{C}$. Soluble material

442 was then were added to NTA nickel resin (400 $\mu \mathrm{L}$ slurry, Qiagen), which was pre-

443 charged (overnight at $4{ }^{\circ} \mathrm{C}$, end-over-end) with recombinant His-tagged GFPnb (12.5

$444 \mu \mathrm{L}, 20 \mathrm{mg} / \mathrm{mL}$ ). This mixture of soluble SMA extracts and charged nickel resin was

445 rotated end-over-end $\left(24 \mathrm{~h}, 4^{\circ} \mathrm{C}\right)$.

447 Nickel resin was pelleted by centrifugation $\left(700 \times g, 2 \mathrm{~min}, 4^{\circ} \mathrm{C}\right)$ and the supernatant 448 fraction was carefully collected. Nickel resin was washed twice with Buffer B and twice 449 with $500 \mu \mathrm{L}$ Wash Buffer [Buffer B containing 20 mM imidazole]. Native nanodiscs

450 bound to His-GFPnb were eluted with Buffer B containing $250 \mathrm{mM}$ imidazole by rotating 451 end-over-end for $20 \mathrm{~min}$ at $4{ }^{\circ} \mathrm{C}$. Due to the presence of GFP nanobody in the elution

452 samples, relative target abundance was determined by Western analysis and anti-GFP

453 band quantification. Protein concentrations of all other samples were quantified by

454 Pierce ${ }^{\mathrm{TM}}$ BCA Protein Assay Kit (Thermo). 
$4581 \times 10^{8}$ yeast cells were harvested by centrifugation $\left(4,000 \mathrm{~g}, 5 \mathrm{~min}, 4^{\circ} \mathrm{C}\right)$. The

459 supernatant was removed, and the cell pellet was flash frozen in $\mathrm{N}_{2}(\mathrm{I})$ and stored at -80

460 C. (I) and stored at $-80^{\circ} \mathrm{C}$. Frozen yeast pellets were thawed on ice and resuspended

461 in $100 \mu \mathrm{L}$ cold water. To this, $100 \mu \mathrm{L}$ of glass beads $(0.5 \mathrm{~mm}$; RPI) and CoQ 10 internal

462 standard $(10 \mu \mathrm{L}, 10 \mu \mathrm{M})$ were added and bead beat $\left(2 \mathrm{~min}, 4^{\circ} \mathrm{C}\right) .900 \mu \mathrm{L}$ extraction

463 solvent $\left(1: 1 \mathrm{CHCl}_{3} / \mathrm{MeOH}, 4^{\circ} \mathrm{C}\right)$ was added and samples were vortexed briefly. To

464 complete phase separation, samples were acidified with $85 \mu \mathrm{L} 6 \mathrm{M} \mathrm{HCl}\left(4^{\circ} \mathrm{C}\right)$, vortexed

$465\left(2 \times 30 \mathrm{~s}, 4^{\circ} \mathrm{C}\right)$, and centrifuged $\left(5,000 \mathrm{~g}, 2 \mathrm{~min}, 4^{\circ} \mathrm{C}\right)$. The resulting aqueous layer

466 (top) was removed and $400 \mu \mathrm{L}$ of the organic layer (bottom) was transferred to a clean

467 tube and dried under $\operatorname{Ar}_{(\mathrm{g})}$. Dried organic matter (lipids) were reconstituted in

$468 \mathrm{ACN} / \mathrm{IPA} / \mathrm{H}_{2} \mathrm{O}(65: 30: 5, \mathrm{v} / \mathrm{v} / \mathrm{v}, 100 \mu \mathrm{L})$ by vortexing $(2 \times 30 \mathrm{~s}, \mathrm{RT})$ and transferred to an

469 amber vial (Sigma; QSertVial ${ }^{\mathrm{TM}}, 12$ x $32 \mathrm{~mm}, 0.3 \mathrm{~mL}$ ) for LC-MS analysis.

471 Petroleum Ether:MeOH Extraction

472 For yeast whole-cell measurements, $1 \times 10^{8}$ cells were collected by centrifugation $(4,000$

$473 \times \mathrm{g}, 5 \mathrm{~min})$ and layered with $100 \mu \mathrm{L}$ of glass beads $(0.5 \mathrm{~mm}$; RPI). Whole-cell samples

474 and all other fractions were then suspended in ice-cold methanol $(500 \mu \mathrm{L}$; with $1 \mu \mathrm{M}$

$475 \mathrm{CoQ}_{8}$ internal standard) and vortexed $\left(10 \mathrm{~min}, 4{ }^{\circ} \mathrm{C}\right) . \sim 500 \mu \mathrm{L}$ of petroleum ether was

476 added to extract lipids, and samples were vortexed $\left(3 \mathrm{~min}, 4^{\circ} \mathrm{C}\right)$ and centrifuged

$477(17,000 \times g, 1 \mathrm{~min})$ to separate phases. The petroleum ether (upper) layer was

478 collected, and the extraction was repeated with another round of petroleum ether (500

$479 \mu \mathrm{L})$, vortexing $\left(3 \mathrm{~min}, 4^{\circ} \mathrm{C}\right)$, and centrifugation $(17,000 \times g, 1 \mathrm{~min})$. The petroleum ether

480 layers were pooled and dried under argon. Lipids were resuspended in 2-propanol (15 
$\mu \mathrm{L}$ ) and transferred to amber glass vials (Sigma; QSertVial ${ }^{\mathrm{TM}}, 12 \times 32 \mathrm{~mm}, 0.3 \mathrm{~mL}$ ).

482 Sodium borohydride (15 $\mu \mathrm{L}$ of $10 \mathrm{mM}$ in 2-propanol) was added to reduce quinones,

483 and samples were vortexed briefly and incubated (5-10 min). Methanol (20 $\mu \mathrm{L})$ was

484 added to remove excess sodium borohydride, and samples were vortexed briefly and

485 incubated (5-10 min). Samples were briefly flushed with nitrogen gas.

\section{Lipidomic Analysis}

489 Targeted LC-MS for Yeast CoQ6 and PPHB 6

490 LC-MS analysis was performed on an Acquity CSH C18 column held at $50{ }^{\circ} \mathrm{C}(100 \mathrm{~mm}$

$491 \times 2.1 \mathrm{~mm} \times 1.7 \mu \mathrm{m}$ particle size; Waters) using a Vanquish Binary Pump (400 $\mu \mathrm{L} / \mathrm{min}$

492 flow rate; Thermo Scientific). Mobile phase A consisted of $10 \mathrm{mM}$ ammonium acetate

493 and $250 \mu \mathrm{L} / \mathrm{L}$ acetic acid in ACN:H2O (70:30, v/v). Mobile phase B consisted of

494 IPA:ACN (90:10, v/v) also with $10 \mathrm{mM}$ ammonium acetate and $250 \mu \mathrm{L} / \mathrm{L}$ acetic acid.

495 Mobile phase B was initially held at $50 \%$ for 1.5 min and then increased to $99 \%$ over 7.5

$496 \mathrm{~min}$ and held there for $2 \mathrm{~min}$. The column was equilibrated for $2.5 \mathrm{~min}$ before the next

497 injection. $10 \mu \mathrm{L}$ of each extract was injected by a Vanquish Split Sampler HT

498 autosampler (Thermo Scientific) in a randomized order.

500 The LC system was coupled to a Q Exactive Orbitrap mass spectrometer (MS) through

501 a heated electrospray ionization (HESI II) source (Thermo Scientific). Source conditions

502 were as follow: HESI II and capillary temperature at $350{ }^{\circ} \mathrm{C}$, sheath gas flow rate at 25

503 units, aux gas flow rate at 15 units, sweep gas flow rate at 5 units, spray voltage at +3.5 
$504 \mathrm{kV} /-3.5 \mathrm{kV}$, and S-lens RF at 90.0 units. The MS was operated in a polarity switching

505 mode acquiring positive and negative full MS and MS2 spectra (Top2) within the same

506 injection. Acquisition parameters for full MS scans in both modes were 17,500

507 resolution, $1 \times 10^{6}$ automatic gain control (AGC) target, $100 \mathrm{~ms}$ ion accumulation time

508 (max IT), and 200 to $1600 \mathrm{~m} / \mathrm{z}$ scan range. MS2 scans in both modes were then

509 performed at 17,500 resolution, $1 \times 10^{5} \mathrm{AGC}$ target, $50 \mathrm{~ms}$ max IT, $1.0 \mathrm{~m} / \mathrm{z}$ isolation

510 window, stepped normalized collision energy (NCE) at 20, 30, 40, and a 10.0 s dynamic

511 exclusion.

512

513 Parallel Reaction Monitoring (PRM) in positive polarity mode was utilized to monitor for

514 two primary adducts, $[\mathrm{M}+\mathrm{H}]^{+}$and $\left[\mathrm{M}+\mathrm{NH}^{4}\right]^{+}$, of each $\mathrm{CoQ}$ species. For $\mathrm{CoQ}_{6}$, we

515 targeted the mass to charge ratio of 592.449 and 609.475 ; for $\mathrm{CoQ}_{8}, 728.574$ and

516 745.601; and for CoQ10, 864.7 and 881.727. PRM MS settings were: Automatic gain

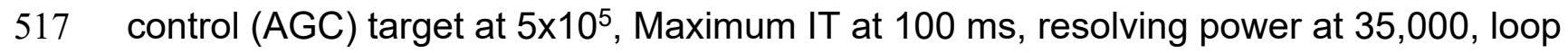

518 count at 2, isolation window at $3.0 \mathrm{~m} / \mathrm{z}$, and collision energy at 35 . Another experiment

519 performed in tandem with PRM used targeted single ion monitoring (t-SIM) in negative

520 mode to determine the primary adduct, $[\mathrm{M}-\mathrm{H}]^{-}$, of $\mathrm{CoQ}$ intermediates. For $\mathrm{PPHB}$, we

521 targeted the mass to charge ratio of 544.908 and used the following t-SIM MS settings:

522 AGC target at $5 \times 10^{5}$, Maximum IT at $100 \mathrm{~ms}$, and resolving power at 140,000 with an

523 isolation window of $4.0 \mathrm{~m} / \mathrm{z}$.

525 Data Analysis 
526 The resulting LC-MS data was manually processed using a custom TraceFinder 4.1

527 (Thermo Scientific) method using a mass precision of 4 and mass tolerance of $10 \mathrm{ppm}$

528 to detect and identify the different species and adducts of $\mathrm{CoQ}_{6}$ and $\mathrm{CoQ}_{8}$ and

529 intermediates.

531 Targeted HPLC-ECD for Yeast CoQ6

532 For yeast whole-cell measurements, $5 \times 10^{8}$ cells were collected by centrifugation $(4,000$

$533 \times \mathrm{g}, 5 \mathrm{~min})$ and layered with $100 \mu \mathrm{L}$ of glass beads (0.5 mm; RPI). Lipids from whole-cell

534 samples and other fractions were extracted according to the "Petroleum Ether:MeOH

535 Extraction" section above. Samples were analyzed by reverse-phase high-pressure

536 liquid chromatography with electrochemical detection (HPLC-ECD) using a C18 column

537 (Thermo Scientific, Betasil C18, $100 \times 2.1 \mathrm{~mm}$, particle size $3 \mu \mathrm{m}$ ) at a flow rate of 0.3

$538 \mathrm{~mL} / \mathrm{min}$ with a mobile phase of $75 \%$ methanol, $20 \% 2$-propanol, and $5 \%$ ammonium

539 acetate $(1 \mathrm{M}, \mathrm{pH} 4.4)$. After separation on the column, the $\mathrm{NaBH}_{4}$-reduced quinones

540 were quantified on ECD detector (Thermo Scientific ECD3000-RS) equipped with

541 6020RS omni Coulometric Guarding Cell "E1", and 6011RS ultra Analytical Cell "E2"

542 and "E3". To prevent premature quinone oxidation, the E1 guarding electrode was set to

$543-200 \mathrm{mV}$. Measurements were made using the analytical E2 electrode operating at 600

$544 \mathrm{mV}$ after complete oxidation of the quinone sample and E3 electrode (600 mV) was

545 used to ensure that total signal was recorded on the E2 cell. For each experiment, a

$546 \mathrm{CoQ}_{6}$ standard in 2-propanol was also prepared with sodium borohydride and methanol

547 treatment, and different volumes were injected to make a standard curve. Quinones 
548 were quantified by integrating respective peaks using the Chromeleon 7.2.10 software

549 (Thermo) and normalized to CoQ8 internal standard.

551 Antibodies and Western Blots

$552 \quad$ Antibodies

553 Primary antibodies used in this study include anti-Kar2 (SCBT sc-33630, 1:5000; RRID:

554 AB_672118), anti-Cit150 (Biomatik, 1:4000), anti- $\beta$-actin (Abcam ab8224, 1:1000; RRID:

555 AB_449644), anti-Tom7051 (1:1000, a gift from Nora Vogtle, University of Freiburg),

556 anti-Vdac (Abcam ab110326, 1:2000; RRID: AB_10865182); anti-GFP (SCBT sc-9996,

557 1:1000; RRID: AB_627695), anti-Sdh2 ${ }^{52}$ (1:5000, a gift from Oleh Khalimonchuk,

558 University of Nebraska). Secondary antibodies include goat anti-mouse (LI-COR 926-

559 32210, 1:15000; RRID: AB_621842) and goat anti-rabbit (LI-COR 926-32211, 1:15000;

560 RRID: AB_621843).

562 SMA Solubility Western Blot

563 Mitoplasts were recovered and solubilized in styrene maleic acid containing buffer as

564 described above in "Native Nanodisc Isolation." To determine the extent of GFP target

565 solubilization, equal amounts of "input" (IP) and soluble supernatant (S) were obtained,

566 along with the total pellet (insoluble, IS). $75 \mu \mathrm{L}$ of the input sample was collected

567 immediately after SMA solubilization. After separating soluble SMA extracts from non-

568 extracted material via centrifugation $\left(21,000 \times \mathrm{g}, 10 \mathrm{~min}, 4^{\circ} \mathrm{C}\right)$, the supernatant was

569 transferred to a clean tube for an additional 5 minute spin. $75 \mu \mathrm{L}$ of soluble sample was

570 then transferred to a new tube. The resulting pellet was washed with $1 \mathrm{~mL}$ of Buffer B 
571 and centrifuged $\left(21,000 \times g\right.$ for $5 \mathrm{~min}$ at $\left.4{ }^{\circ} \mathrm{C}\right)$. The resulting supernatant was aspirated

572 and $75 \mu \mathrm{L}$ of Buffer B was added to the insoluble (IS) fraction. From each sample,

573 proteins were extracted by standard chloroform-methanol procedures. Precipitated

574 protein was reconstituted in $75 \mu \mathrm{L} 0.1 \mathrm{M} \mathrm{NaOH} .25 \mu \mathrm{L} 4 \mathrm{X}$ LDS sample buffer containing

575 beta-mercaptoethanol (BME) was added and samples were incubated (95 $\left.{ }^{\circ} \mathrm{C}, \sim 10 \mathrm{~min}\right)$.

576 Proteins were analyzed with 4-12\% Novex NuPAGE Bis-Tris SDS-PAGE (Invitrogen)

577 gels $(1 \mathrm{~h}, 150 \mathrm{~V})$. The gel was transferred to PVDF membrane at $100 \mathrm{~V}$ for $1 \mathrm{~h}$ with

578 transfer buffer (192 mM glycine, $25 \mathrm{mM}$ Tris, 20\% methanol [v/v]). The membrane was

579 blocked with 5\% nonfat dry milk (NFDM) in TBST (20 mM Tris pH 7.4, $150 \mathrm{mM} \mathrm{NaCl,}$

$580 \quad 0.05 \%$ Tween $20[\mathrm{v} / \mathrm{v}])(1 \mathrm{~h}$ with agitation). Antibodies were diluted in $1 \%$ NFDM in

581 TBST and incubated with the PVDF (overnight, $4^{\circ} \mathrm{C}$ with agitation). The PVDF was

582 washed three times in TBST and the secondary antibodies were diluted 1:15,000 in $1 \%$

583 NFDM in TBST (1.5 h, r.t.). The membrane was washed three times in TBST and

584 imaged on a LI-COR Odyessey CLx using Image Studio v5.2 software.

586 SMALP Fractionation Western Blot

587 Fractions described above in "Native Nanodisc Isolation" and "SMA Solubility Western

588 Blot" were collected and used for western blot analysis. $4 \mu \mathrm{g}$ of spheroplasts (SP) and

589 crude mitochondria (CM) were loaded, along with equal volumes of extracted soluble

590 (S) and final elution (E) samples. Western blots were performed as described above. 
593 All experiments were performed in at least biological triplicate, unless stated otherwise.

594 In all cases, "mean" refers to arithmetic mean, and "SD" refers to sample standard

595 deviation. Statistical analyses were performed using Microsoft Excel. $p$-values were

596 calculated using an unpaired, two-tailed, Student's $t$-test. In all cases, $n$ represents

597 independent replicates of an experiment.

598

599 Reporting Summary

600 Further information on research design is available in the Nature Research Reporting

601 Summary linked to this article.

602

603

604 Data availability

605 Next generation sequencing data (Fig. 2, Extended Data Fig 1) have been deposited to

606 NCBI SRA (BioProject ID PRJNA679831; SRA accession SRP293543). Additional

607 source data for Fig. 1-4 and Extended Data 1-3 are provided with the paper. All other

608 data supporting the finding of this study are available from the corresponding authors on

609 reasonable request.

610

611 


\section{References}

613 1. Hatefi, Y., Haavik, A.G., Fowler, L.R. \& Griffiths, D.E. Studies on the electron

614 transfer system. XLII. Reconstitution of the electron transfer system. J Biol Chem

$615 \quad 237,2661-2669(1962)$.

616 2. Frerman, F.E. Acyl-CoA dehydrogenases, electron transfer flavoprotein and

617 electron transfer flavoprotein dehydrogenase. Biochem Soc Trans 16, 416-418

$618 \quad$ (1988).

619 3. Jones, M.E. Pyrimidine nucleotide biosynthesis in animals: genes, enzymes, and 620 regulation of UMP biosynthesis. Annu Rev Biochem 49, 253-279 (1980).

621 4. Bentinger, M., Brismar, K. \& Dallner, G. The antioxidant role of coenzyme Q.

$622 \quad$ Mitochondrion 7 Suppl, S41-50 (2007).

623 5. Do, T.Q., Schultz, J.R. \& Clarke, C.F. Enhanced sensitivity of ubiquinonedeficient mutants of Saccharomyces cerevisiae to products of autoxidized polyunsaturated fatty acids. Proc Natl Acad Sci U S A 93, 7534-7539 (1996).

6. Avery, A.M. \& Avery, S.V. Saccharomyces cerevisiae expresses three phospholipid hydroperoxide glutathione peroxidases. J Biol Chem 276, 33730-

629 7. Choi, Y., Sims, G.E., Murphy, S., Miller, J.R. \& Chan, A.P. Predicting the functional effect of amino acid substitutions and indels. PLoS One 7, e46688 (2012).

632 8. Tauche, A., Krause-Buchholz, U. \& Rodel, G. Ubiquinone biosynthesis in Saccharomyces cerevisiae: the molecular organization of O-methylase Coq3p depends on Abc1p/Coq8p. FEMS Yeast Res 8, 1263-1275 (2008). 
635 9. Vogtle, F.N. et al. Landscape of submitochondrial protein distribution. Nat Commun 8, 290 (2017).

637 10. Rhee, H.W. et al. Proteomic mapping of mitochondria in living cells via spatially 638 restricted enzymatic tagging. Science 339, 1328-1331 (2013).

639 11. Stefely, J.A. et al. Cerebellar Ataxia and Coenzyme Q Deficiency through Loss of $640 \quad$ Unorthodox Kinase Activity. Mol Cell 63, 608-620 (2016).

641 12. Reidenbach, A.G. et al. Conserved Lipid and Small-Molecule Modulation of COQ8 Reveals Regulation of the Ancient Kinase-like UbiB Family. Cell Chem Biol 25, 154-165 e111 (2018).

644 13. Morgenstern, M. et al. Definition of a High-Confidence Mitochondrial Proteome at 645 Quantitative Scale. Cell Rep 19, 2836-2852 (2017).

646 14. Vazquez-Fonseca, L. et al. ADCK2 Haploinsufficiency Reduces Mitochondrial Lipid Oxidation and Causes Myopathy Associated with CoQ Deficiency. J Clin

649 15. Stefely, J.A. et al. Mitochondrial ADCK3 employs an atypical protein kinase-like 650 fold to enable coenzyme Q biosynthesis. Mol Cell 57, 83-94 (2015).

651 16. Kannan, N., Taylor, S.S., Zhai, Y., Venter, J.C. \& Manning, G. Structural and 652 functional diversity of the microbial kinome. PLoS Biol 5, e17 (2007).

653 17. Tan, T., Ozbalci, C., Brugger, B., Rapaport, D. \& Dimmer, K.S. Mcp1 and Mcp2, 654 two novel proteins involved in mitochondrial lipid homeostasis. J Cell Sci 126, 3563-3574 (2013). 
656 18. Odendall, F. et al. The mitochondrial intermembrane space-facing proteins Mcp2 and Tgl2 are involved in yeast lipid metabolism. Mol Biol Cell 30, 2681-2694 (2019).

659 19. Costanzo, M. et al. A global genetic interaction network maps a wiring diagram of cellular function. Science 353 (2016).

661 20. Lee, S.C. et al. A method for detergent-free isolation of membrane proteins in their local lipid environment. Nat Protoc 11, 1149-1162 (2016).

663 21. Oyedotun, K.S. \& Lemire, B.D. The Quinone-binding sites of the Saccharomyces cerevisiae succinate-ubiquinone oxidoreductase. J Biol Chem 276, 16936-16943 (2001).

666 22. Murley, A. \& Nunnari, J. The Emerging Network of Mitochondria-Organelle Contacts. Mol Cell 61, 648-653 (2016).

668 23. Tamura, Y., Kawano, S. \& Endo, T. Organelle contact zones as sites for lipid transfer. J Biochem 165, 115-123 (2019).

670 24. Subramanian, K. et al. Coenzyme Q biosynthetic proteins assemble in a substrate-dependent manner into domains at ER-mitochondria contacts. J Cell Biol 218, 1353-1369 (2019).

673 25. Eisenberg-Bord, M. et al. The Endoplasmic Reticulum-Mitochondria Encounter Structure Complex Coordinates Coenzyme Q Biosynthesis. Contact (Thousand Oaks) 2, 2515256418825409 (2019).

676 26. Tirrell, P.S., Nguyen, K.N., Luby-Phelps, K. \& Friedman, J.R. MICOS subcomplexes assemble independently on the mitochondrial inner membrane in proximity to ER contact sites. J Cell Biol 219 (2020). 
679 27. Lohman, D.C. et al. An Isoprene Lipid-Binding Protein Promotes Eukaryotic

$680 \quad$ Coenzyme Q Biosynthesis. Mol Cell 73, 763-774 e710 (2019).

681 28. Hoppins, S. et al. A mitochondrial-focused genetic interaction map reveals a 682 scaffold-like complex required for inner membrane organization in mitochondria.

$683 \quad$ J Cell Biol 195, 323-340 (2011).

684 29. Leonard, C.J., Aravind, L. \& Koonin, E.V. Novel families of putative protein kinases in bacteria and archaea: evolution of the "eukaryotic" protein kinase

30. Lundquist, P.K., Davis, J.I. \& van Wijk, K.J. ABC1K atypical kinases in plants: filling the organellar kinase void. Trends Plant Sci 17, 546-555 (2012).

689 31. Pralon, T. et al. Mutation of the Atypical Kinase ABC1K3 Partially Rescues the PROTON GRADIENT REGULATION 6 Phenotype in Arabidopsis thaliana. Front Plant Sci 11, 337 (2020).

32. Ashraf, S. et al. ADCK4 mutations promote steroid-resistant nephrotic syndrome through CoQ10 biosynthesis disruption. J Clin Invest 123, 5179-5189 (2013).

694 33. Lagier-Tourenne, C. et al. ADCK3, an ancestral kinase, is mutated in a form of recessive ataxia associated with coenzyme Q10 deficiency. Am J Hum Genet 82, 661-672 (2008).

697 34. Wiedemeyer, W.R. et al. Pattern of retinoblastoma pathway inactivation dictates response to CDK4/6 inhibition in GBM. Proc Natl Acad Sci U S A 107, 1150111506 (2010).

35. Brough, R. et al. Functional viability profiles of breast cancer. Cancer Discov 1, 260-273 (2011). 
702 36. Simpson, K.J. et al. Identification of genes that regulate epithelial cell migration using an siRNA screening approach. Nat Cell Biol 10, 1027-1038 (2008).

704 37. Qiu, M. et al. aarF domain containing kinase 5 gene promotes invasion and migration of lung cancer cells through ADCK5-SOX9-PTTG1 pathway. Exp Cell Res 392, 112002 (2020).

38. Bersuker, K. et al. The CoQ oxidoreductase FSP1 acts parallel to GPX4 to inhibit ferroptosis. Nature 575, 688-692 (2019).

39. Doll, S. et al. FSP1 is a glutathione-independent ferroptosis suppressor. Nature 575, 693-698 (2019).

711 40. Asquith, C.R.M., Murray, N.H. \& Pagliarini, D.J. ADCK3/COQ8A: the choice target of the UbiB protein kinase-like family. Nat Rev Drug Discov 18, 815 (2019).

713 41. Huh, W.K. et al. Global analysis of protein localization in budding yeast. Nature 425, 686-691 (2003).

42. Baudin, A., Ozier-Kalogeropoulos, O., Denouel, A., Lacroute, F. \& Cullin, C. A simple and efficient method for direct gene deletion in Saccharomyces

43. Generoso, W.C., Gottardi, M., Oreb, M. \& Boles, E. Simplified CRISPR-Cas genome editing for Saccharomyces cerevisiae. J Microbiol Methods 127, 203-

721 44. Gietz, R.D. \& Woods, R.A. Transformation of yeast by lithium acetate/single(2002). 
724 45. Yang, J. et al. The I-TASSER Suite: protein structure and function prediction. Nat

$725 \quad$ Methods 12, 7-8 (2015).

726 46. Meisinger, C., Pfanner, N. \& Truscott, K.N. Isolation of yeast mitochondria.

$727 \quad$ Methods Mol Biol 313, 33-39 (2006).

728 47. Forner, F., Arriaga, E.A. \& Mann, M. Mild protease treatment as a small-scale

biochemical method for mitochondria purification and proteomic mapping of

cytoplasm-exposed mitochondrial proteins. J Proteome Res 5, 3277-3287 (2006).

48. Fox, B.G. \& Blommel, P.G. Autoinduction of protein expression. Curr Protoc Protein Sci Chapter 5, Unit 523 (2009).

734 49. Niesen, F.H., Berglund, H. \& Vedadi, M. The use of differential scanning fluorimetry to detect ligand interactions that promote protein stability. Nat Protoc 2, 2212-2221 (2007).

737 50. Guo, X. et al. Ptc7p Dephosphorylates Select Mitochondrial Proteins to Enhance

51. Vogtle, F.N. et al. Mutations in PMPCB Encoding the Catalytic Subunit of the

742 52. Bohovych, I. et al. Metalloprotease OMA1 Fine-tunes Mitochondrial Bioenergetic Function and Respiratory Supercomplex Stability. Sci Rep 5, 13989 (2015). 


\section{Acknowledgements}

747 We thank Steven Claypool for consultation on SMALP generation, Adam Frost for

748 providing the plasmid containing GFP nanobody, Nora Vogtle and Oleh Khalimonchuk

749 for providing mitochondrial antibodies, Jared Rutter and Jodi Nunnari for providing the

750 parental yeast strains used in our studies, Matt Stefely for assistance with figure

751 generation, and current and former members of the Pagliarini Laboratory for their

752 feedback. This work was supported by NIH R35GM131795 and R01 GM112057 (to

753 D.J.P.), NIH T32DK007665, William H. Peterson Fellowship, Washburn Wharton

754 Fellowship, and University of Wisconsin Biochemistry Funding (to Z.A.K.), a National

755 Science Foundation Graduate Research Fellowship DGE-1747503 (to K.P.R.), and

756 P41GM108538 (to J.J.C. and D.J.P.).

\section{Author information}

759 These authors contributed equally: Zachary A. Kemmerer, Kyle P. Robinson.

761 Affiliations

762 Morgridge Institute for Research, Madison, WI, USA.

763 Zachary A. Kemmerer, Kyle P. Robinson, Jonathan M. Schmitz, Adam Jochem, and

764 David J. Pagliarini

766 Department of Biochemistry, University of Wisconsin-Madison, Madison, WI, USA.

767 Zachary A. Kemmerer, Kyle P. Robinson, Jonathan M. Schmitz, Adam Jochem, and

768 David J. Pagliarini 
770 Genome Center of Wisconsin, Madison, Wisconsin, USA.

771 Joshua J. Coon

772

773 Department of Chemistry, University of Wisconsin-Madison, Madison, WI, USA.

774 Brett R. Paulson, Paul D. Hutchins, and Joshua J. Coon

775

776 Department of Biomolecular Chemistry, University of Wisconsin-Madison, Madison, WI,

777 USA.

778 Joshua J. Coon

779

780 Departments of Cell Biology and Physiology; Biochemistry and Molecular Biophysics;

781 and Genetics, Washington University School of Medicine, St. Louis, MO, USA

782 David J. Pagliarini

783

784 Contributions

785 Z.A.K., K.P.R., and D.J.P. conceived of the project and its design. Z.A.K. and K.P.R.

786 conducted experiments and performed data analysis. J.M.S. purified and characterized

787 GFP nanobody. B.R.P. and P.D.H. performed and analyzed mass spectrometry

788 experiments. A.J. contributed to new reagents (cloning). All authors edited the

789 manuscript. Z.A.K., K.P.R., and D.J.P. wrote the manuscript. D.J.P. supervised the

790 project.

791

792 


\section{Corresponding Author}

794 Correspondence and requests for materials should be addressed to D.J.P.

795 (pagliarini@wustl.edu).

797 Ethics declaration

798 Competing interests

799 J.J.C. is a consultant for Thermo Fisher Scientific.

\section{Extended data}

a

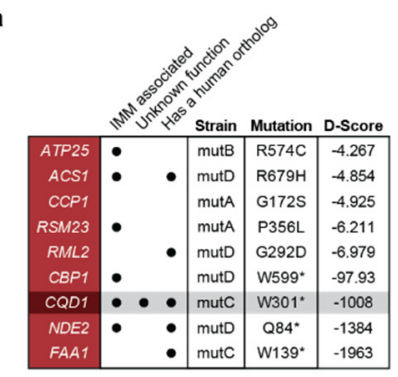

b

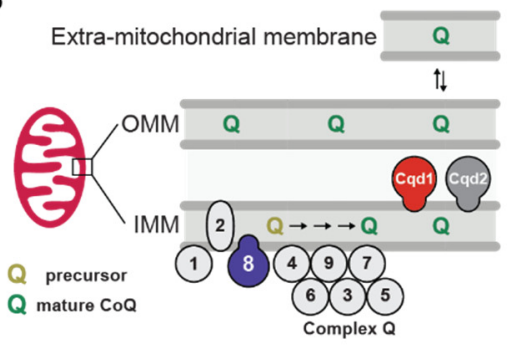

c

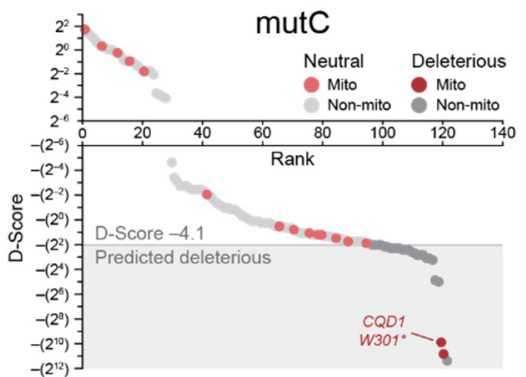

Extended Data Fig. 1 Genome-wide screen for CoQ trafficking genes identifies uncharacterized UbiB protein Cqd1. a, Criteria for nine mitochondrial candidates used to nominate genes for additional investigation. Submitochondrial localization was confirmed by comparison to previous submitochondrial profiling datasets ${ }^{9}$, while protein function and human ortholog criteria were determined with existing database mining (UniProt and PhylomeDB, respectively). b, Schematic showing the submitochondrial localization of UbiB family proteins Coq8 (purple), Cqd1 (red), and Cqd2 (gray). Coq8 is essential for CoQ biosynthesis in concert with other Coq proteins (Coq1-9). c, Non-synonymous mutations identified using whole-genome sequencing for strain mutC were analyzed with PROVEAN to filter for likely deleterious changes (D-score $\leq-4.1$, shaded box). Gray, all genes; red, mitochondrial genes. Light, predicted neutral; dark, predicted deleterious. 
bioRxiv preprint doi: https://doi.org/10.1101/2020.12.09.418202; this version posted December 9, 2020. The copyright holder for this preprint (which was not certified by peer review) is the author/funder, who has granted bioRxiv a license to display the preprint in perpetuity. It is made available under aCC-BY-NC-ND 4.0 International license.

a

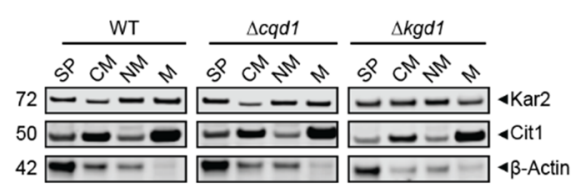

b

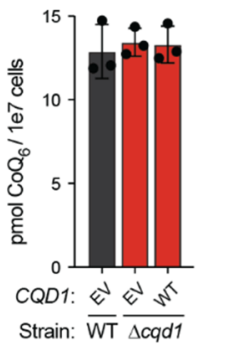

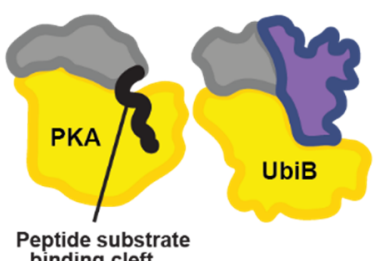

Peptide substrate
binding cleft

d

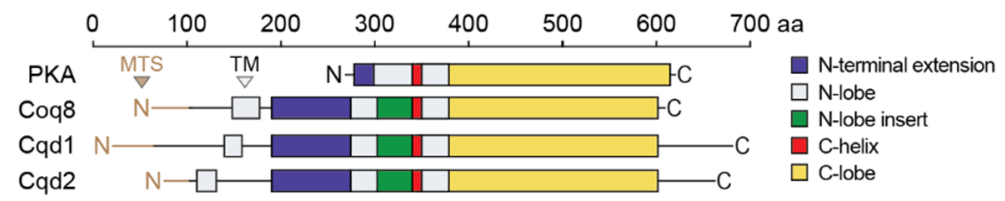

e

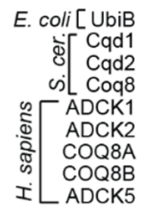

(1) (2)

WIKFGQML

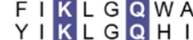

A

$F \mid K \vee G Q H L$

$Y$ I $K$ K I $G$ G $Q W A$

$A L K V G Q M$

$Y \vee K L G Q Q$

KxGQ motif
(3)(4)

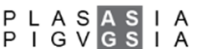

$P$ I GVASLA

PMAAASIG

$P\llcorner G T$

PVGS

P PAAASI G

$\begin{array}{llll}P & \text { F A A S S I G } \\ P \text { I A A A S S L A } & \end{array}$

A-rich loop

UbiB-specific features

Invariant UbiB motif within domain occluding

Residue protein active site

Function K binds ATP $\mathrm{y}$-phosphate upon nucleotide binding

$Q$ forms salt bridge to "releasing" enzyme inhibition

PKA
Residue

NA

f

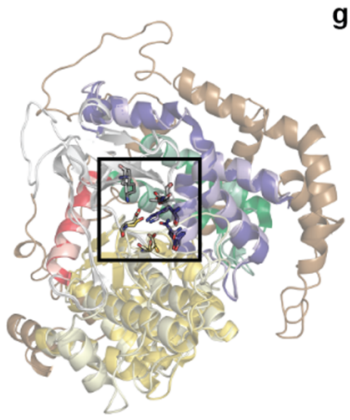

For ePKs, this is "G-rich" loop.

Coordinates ATP $Y$-phosphate

For UbiB, loop may dictate nucleotide specificity

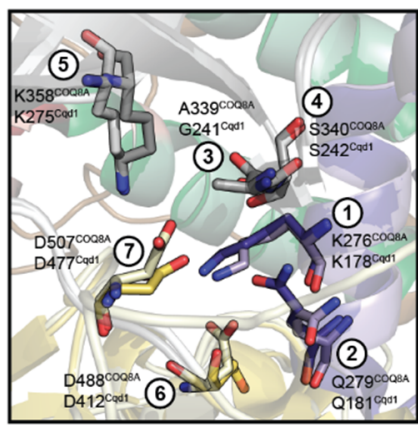

conserved glutamate
(5)

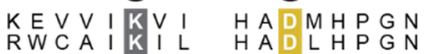

$\begin{array}{lll}R W C A I K I L & H A D L H P G N \\ \text { SSVAVKC }\end{array}$

QRVVVKIQ $\mathrm{QTDPHGGN}$

RTVAVKVQ HCDPHPGN

I SVAVKVL HADLHPGN

RE

$T S V A \vee K V Q \quad H S D P H P G N$

HxDxxxxN motif
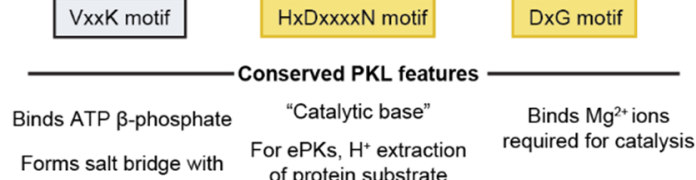
of protein substrate

For UbiB, inhibitory

salt bridge until

nucleotide binds

$$
\text { D166 }
$$

D184

h

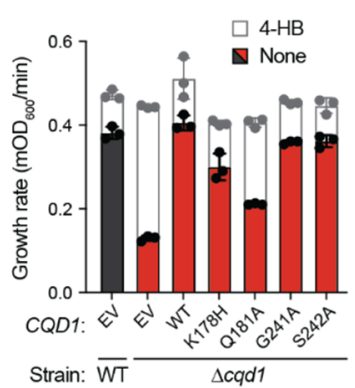


806 Extended Data Fig. 2 Cqd1 influences cellular CoQ distribution. a, Western blot of subcellular samples derived from fractionated WT, $\Delta c q d 1$, and $\Delta k g d 1$ yeast. Spheroplast, SP; crude mitochondria, CM; nonmitochondrial fraction, NM; enriched mitochondria, M. Kar2, endoplasmic reticulum; Cit1, mitochondria; $\beta$-actin, cytoplasm. b. Total CoQ from WT and $\Delta c q d 1$ yeast transformed with the indicated plasmids and grown in Ura-, pABA- media containing $0.1 \%(w / v)$ glucose and $3 \%(w / v)$ glycerol (mean $\pm S D, n=3)$. c, Cartoon of canonical protein kinase A (PKA; 1ATP) and human COQ8A (4PED) showing protein domain organization. Protein kinases often contain a $\beta$-sheet rich $\mathrm{N}$-terminal domain (gray) and a helical $\mathrm{C}$-terminal domain (yellow). COQ8A contains a unique N-terminal extension (purple) containing the invariant UbiB-specific 'KxGQ' motif. d, Domain alignment of PKA and yeast UbiB proteins. Mitochondrial targeting sequence, MTS; transmembrane domain, TM. e, UbiB family sequence alignment of $\mathrm{UbiB}$-specific and conserved protein kinase-like (PKL) features. Residue functions within the canonical protein kinase or UbiB architecture are described below. The three conserved PKL residues shown are essential for phosphoryl transfer activity. f, Homology model for Cqd1 (light) aligned with COQ8A (4PED, dark). The model was threaded using I-TASSER ${ }^{45}$ and COQ8A structure to guide modeling. Boxed and outlined in black are residues described in $\mathbf{g}$, and unmodeled regions are colored in brown. $\mathbf{i}$, Zoomed in view of conserved PKL and UbiB-specific residues. $\mathbf{h}$, Growth rate of WT and $\Delta c q d 1$ yeast transformed with the indicated plasmids (EV, CQD1 or CQD1 point mutants) and grown in Ura-, pABA- media containing $0.1 \%(\mathrm{w} / \mathrm{v})$ glucose and $3 \%(w / v)$ glycerol (mean $\pm S D, n=3$ ). Yeast were treated with 0 (colored bars) or $1 \mu \mathrm{M} 4-\mathrm{HB}$ (white bars, superimposed) to determine rescue of respiratory growth. 
a

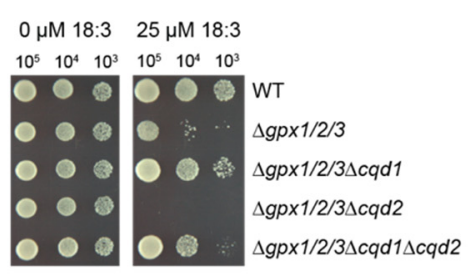

C
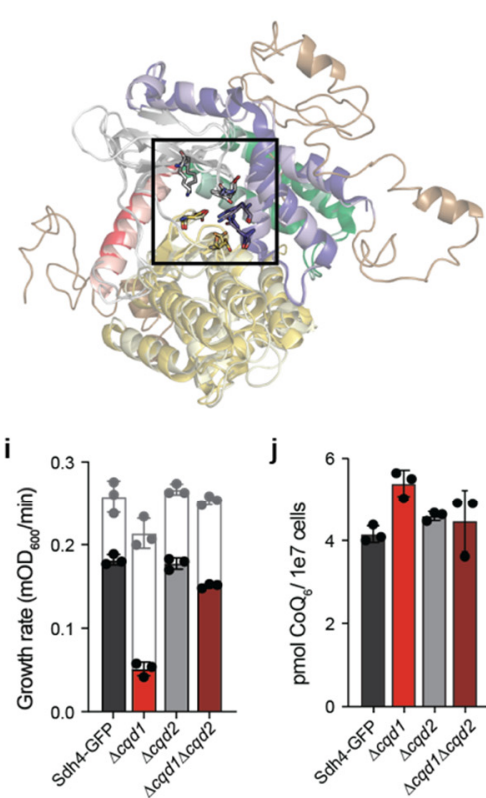

k

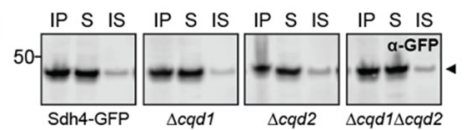

b

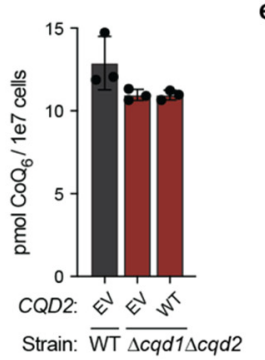

d

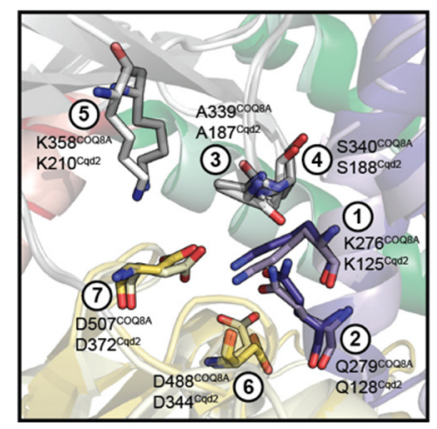

e
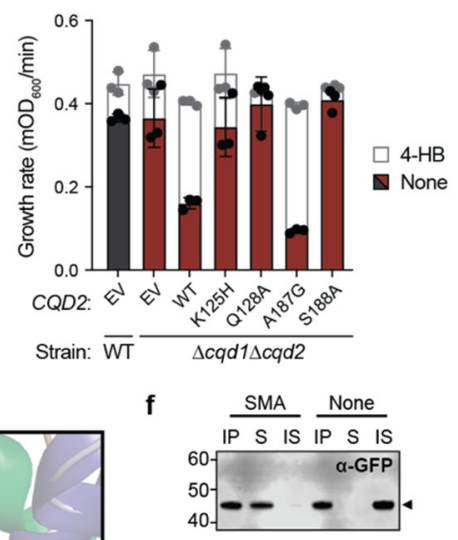

g

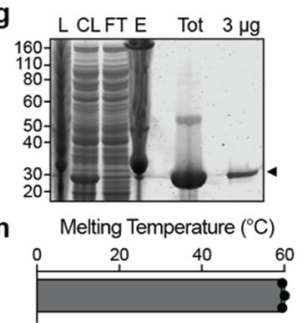

807

Extended Data Fig. 3 Cqd2 function opposes Cqd1 control of CoQ distribution.

a, Serial dilution drop assay of indicated yeast strains grown for 3 days on solid $p A B A-$ medium containing $2 \%$ $(\mathrm{w} / \mathrm{v})$ glucose, $0.5 \%(\mathrm{w} / \mathrm{v})$ ethanol $(\mathrm{EtOH})$, and 0-25 $\mu \mathrm{M}$ 18:3. b. Total CoQ from WT and $\Delta c q d 1 \Delta c q d 2$ yeast transformed with EV or endogenous CQD2 and grown in Ura-, pABA- media containing $0.1 \%(\mathrm{w} / \mathrm{v})$ glucose and $3 \%(w / v)$ glycerol (mean $\pm S D, n=3$ ). c, Homology model for Cqd2 (light) aligned with COQ8A (4PED, dark). The model was threaded using I-TASSER ${ }^{45}$ and COQ8A structure to guide modeling. Boxed and outlined in black are residues described previously (Extended Data Fig. 2f) and unmodeled regions are colored in brown. d, Zoomed in view of conserved PKL and UbiB-specific residues described previously. e, Growth rate of WT and $\Delta c q d 1 \Delta c q d 2$ yeast transformed with the indicated plasmids and grown in Ura-, pABA- media containing $0.1 \%$ $(\mathrm{w} / \mathrm{v})$ glucose and $3 \%(\mathrm{w} / \mathrm{v})$ glycerol (mean $\pm \mathrm{SD}, \mathrm{n}=3$ ). Yeast were treated with 0 (colored bars) or $1 \mu \mathrm{M} 4-\mathrm{HB}$ (white bars, superimposed) to determine recapitulation of respiratory growth defect. f, Western blot to determine

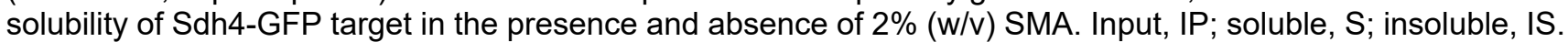
g, Recombinant purification of His-tagged GFP nanobody (GFPnb) via nickel resin enrichment and sizeexclusion isolation. Lysate, L; clarified lysate, CL; flow-through, FT; elution, E; size-exclusion chromatography, SEC. $\mathbf{h}$, Differential scanning fluorimetry of recombinant GFPnb to determine protein melting temperature. $\mathbf{i}$, Growth rate of Sdh4-GFP yeast and indicated deletion strains assayed in pABA- media containing $0.1 \%(\mathrm{w} / \mathrm{v})$ glucose and $3 \%(\mathrm{w} / \mathrm{v})$ glycerol and treated with 0 (colored bars) or $1 \mu \mathrm{M} 4-\mathrm{HB}$ (white bars, superimposed) (mean $\pm S D, n=3$ ). j, Total CoQ from yeast strains described in $\mathbf{i}$. $\mathbf{k}$, Western blot to determine solubility of Sdh4-GFP target during SMALP preparation from the indicated yeast strains. Input, IP; soluble, S; insoluble, IS. I, Western blot of SMALP isolation samples derived from the indicated yeast. Spheroplast, SP; crude mitochondria, CM; soluble, S; elution, elution, E (or IMM patch). Kar2, endoplasmic reticulum; Tom70, OMM; Vdac, OMM; Sdh4GFP, SMALP target/IMM; Sdh2, IMM; Cit1, mitochondrial matrix. 